\title{
INSIGHTS ON THE DUSTY TORUS AND NEUTRAL TORUS FROM OPTICAL AND X-RAY OBSCURATION IN A COMPLETE VOLUME LIMITED HARD X-RAY AGN SAMPLE
}

\author{
R. I. Davies ${ }^{1}$, L. Burtscher ${ }^{1}$, D. Rosario ${ }^{1}$, T. Storchi-Bergmann ${ }^{2}$, A. Contursi ${ }^{1}$, R. Genzel ${ }^{1}$, J. Graciá- Carpio ${ }^{1}$, E. Hicks ${ }^{3}$,

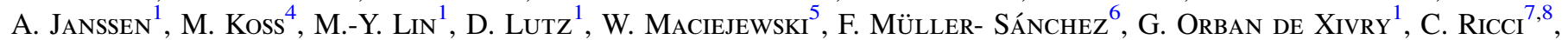 \\ R. Riffel ${ }^{2}$, R. A. Riffel ${ }^{9}$, M. Schartmann ${ }^{10}$, A. Schnorr- Müller ${ }^{1}$, A. Sternberg ${ }^{11}$, E. Sturm ${ }^{1}$, \\ L. TACCONI ${ }^{1}$, AND S. VEILleuX ${ }^{12}$ \\ ${ }^{1}$ Max-Planck-Institut für extraterrestrische Physik, Postfach 1312, D-85741, Garching, Germany \\ ${ }^{2}$ Departamento de Astronomia, Universidade Federal do Rio Grande do Sul, IF, CP 15051, 91501-970 Porto Alegre, RS, Brazil \\ ${ }_{3}^{3}$ Astronomy Department, University of Alaska, Anchorage, USA \\ ${ }^{4}$ Institute for Astronomy, Department of Physics, ETH Zurich, Wolfgang-Pauli-Strasse 27, CH-8093 Zurich, Switzerland \\ ${ }^{5}$ Astrophysics Research Institute, Liverpool John Moores University, IC2 Liverpool Science Park, 146 Brownlow Hill, L3 5RF, UK \\ ${ }^{6}$ Center for Astrophysics and Space Astronomy, University of Colorado, Boulder, CO 80309-0389, USA \\ 7 Pontificia Universidad Católica de Chile, Instituto de Astrofísica, Casilla 306, Santiago 22, Chile \\ ${ }^{8}$ EMBIGGEN Anillo, Concepción, Chile \\ ${ }^{9}$ Departamento de Física, Centro de Ciências Naturais e Exatas, Universidade Federal de Santa Maria, 97105-900 Santa Maria, RS, Brazil \\ ${ }^{10}$ Centre for Astrophysics and Supercomputing, Swinburne University of Technology, Hawthorn, Victoria, 3122, Australia \\ ${ }^{11}$ Raymond and Beverly Sackler School of Physics \& Astronomy, Tel Aviv University, Ramat Aviv 69978, Israel \\ ${ }^{12}$ Department of Astronomy and Joint Space-Science Institute, University of Maryland, College Park, MD 20742-2421 USA \\ Received 2015 February 10; accepted 2015 May 3; published 2015 June 12
}

\begin{abstract}
We describe a complete volume limited sample of nearby active galaxies selected by their 14-195 keV luminosity, and outline its rationale for studying the mechanisms regulating gas inflow and outflow. We also describe a complementary sample of inactive galaxies, selected to match the host galaxy properties. The active sample appears to have no bias in terms of active galactic nucleus (AGN) type, the only difference being the neutral absorbing column, which is two orders of magnitude greater for the Seyfert $2 \mathrm{~s}$. In the luminosity range spanned by the sample, $\log L_{14-195 \mathrm{keV}}\left[\mathrm{erg} \mathrm{s}^{-1}\right]=42.4-43.7$, the optically obscured and X-ray absorbed fractions are 50\%$65 \%$. The similarity of these fractions to more distant spectroscopic AGN samples, although over a limited luminosity range, suggests that the torus does not strongly evolve with redshift. Our sample confirms that X-ray unabsorbed Seyfert 2s are rare, comprising not more than a few percent of the Seyfert 2 population. At higher luminosities, the optically obscured fraction decreases (as expected for the increasing dust sublimation radius), but the X-ray absorbed fraction changes little. We argue that the cold X-ray absorption in these Seyfert 1 s can be accounted for by neutral gas in clouds that also contribute to the broad-line region (BLR) emission, and suggest that a geometrically thick neutral gas torus co-exists with the BLR and bridges the gap to the dusty torus.
\end{abstract}

Key words: galaxies: active - galaxies: nuclei - galaxies: Seyfert - X-rays: galaxies

\section{INTRODUCTION}

Feeding and feedback have become a paradigm of galaxy evolution models; by quenching star formation, feedback from active galactic nuclei (AGNs) is thought to shape the galaxy luminosity function and create the bi-modal color sequence in galaxy populations (Benson et al. 2003; Kauffmann et al. 2003). However, the prescriptions used in models (Springel et al. 2005; Croton et al. 2006; Somerville et al. 2008) are relatively simple because observations have focussed on the question of where the inflowing material originates (mergers versus secular evolution) and on integrated galaxy properties. To redress this, in addition to large-scale data, we need also to understand how the material flows toward the black hole $(\mathrm{BH})$ on smaller scales. However these scales cannot be spatially resolved at $z>1$ where co-evolution largely occurs (Fabian 2012). Instead, it is local galaxies that currently offer the only opportunity to guide the small-scale aspects of galaxy and BH co-evolution models. That many local AGNs-in particular Seyferts-are disky systems does not necessarily reduce their validity as templates for more distant galaxies. The nested simulations of Hopkins \& Quataert (2010) provide an important insight in this respect; they suggest that as one looks further inside the central kiloparsec, disk processes become increasingly important in driving gas inwards, independent of what has occurred on large scales. Observations also appear to confirm that, even at $z=1-2$, disk processes are a major contributor to AGN fueling (Kocevski et al. 2012; Schawinski et al. 2012; Karouzos et al. 2014; Villforth et al. 2014).

In nearby archetypal objects, integral field spectroscopy (at optical and infrared wavelengths, and sometimes with adaptive optics) has probed kinematics on 10-100 pc scales, leading to insights in both inflow (see Storchi-Bergmann 2014) and outflow (see Storchi-Bergmann 2015). Despite the inherent complexities of performing detailed systematic studies with such techniques and the large amount of observing time needed, there has been some progress using integral field spectroscopy for very small samples of AGNs (Sosa-Brito et al. 2001; Barbosa et al. 2006, 2009; Davies et al. 2007; Hicks et al. 2009; Müller-Sánchez et al. 2011; Rupke \& Veilleux 2011, 2013, F. Müller-Sánchez et al. 2015, in preparation), and in a few cases with a matched sample of inactive galaxies for comparison (Dumas et al. 2007; Westoby et al. 2012; Hicks et al. 2013; Davies et al. 2014). There are also two larger studies of local AGNs based on integral field spectroscopy, but these do not address spatially resolved structures or kinematics. Burtscher et al. (2015) focus on the spatially unresolved near- 
infrared non-stellar continuum in Seyferts and other low luminosity AGNs (using the spatial information only to help characterize this component); Sturm et al. (2011) and Veilleux et al. (2013) focus on ULIRGs and QSOs, for which the data are spatially unresolved due to the long wavelengths observed.

In this paper, we present a complete, volume limited sample of nearby bright hard X-ray selected AGNs, in a luminosity range that overlaps with AGNs at higher redshift. This statistically meaningful sample of 20 AGNs is complemented by a sample of inactive galaxies that are matched in mass, morphology, and inclination. In Section 2 we describe the scientific rationale for, and the selection of, both the active and inactive samples, and outline the observations that have been started in order to spatially and spectrally resolve the processes that drive and regulate gas inflow and outflow on small scales. We then look at the AGN properties in Section 3, in particular the fraction of Sy $1 \mathrm{~s}$ and Sy $2 \mathrm{~s}$, and the neutral gas columns. Reconciling this with the numbers reported in the literature leads to a discussion of optical obscuration and X-ray absorption. Section 4 focusses on the issue of X-ray unabsorbed Sy 2s. Then in Section 5 we look at what the differing luminosity dependencies of the optically obscured and $\mathrm{X}$-ray absorbed fractions can tell us about the torus and broadline region (BLR). We have chosen this approach-avoiding the need to invoke torus models-because current torus models are in need of additional basic constraints. As pointed out by Hönig (2013) and Feltre et al. (2012), different models can come to contradictory conclusions and model parameters are often degenerate.

\section{SAMPLE}

Most of the studies in the literature that focus on the structure and processes occurring in the circumnuclear regions of samples of local AGNs, have selected objects using the optical classification (e.g., as Seyfert or LINER) as the only defining characteristic of the AGNs. An obvious reason for doing so is that there is a wide variety of local AGNs, and such studies often try to span a range in AGN types. However, this may lead to confusing results because of the impact of the accretion rate, or equivalently and as a proxy for it, luminosity (Ho 2008; Davies et al. 2012; Kormendy \& Ho 2013). For example, a bright Seyfert such as NGC 1068 with $\log L_{\mathrm{AGN}} \sim 45^{13}$ requires an accretion rate of order $0.1 M_{\odot} \mathrm{yr}^{-1}$, while a low-luminosity AGN such as M81 with $\log L_{\mathrm{AGN}} \sim 41$ will have an accretion rate $\sim 10^{-5} M_{\odot} \mathrm{yr}^{-1}$ that is 4 orders of magnitude lower. This difference has a dramatic impact on fuelling requirements. Hicks et al. (2009) and Mazzalay et al. (2013) show that one can expect to find a gas mass of order $10^{7} M_{\odot}$ within a radial scale of $\sim 10 \mathrm{pc}$. Assuming a $1 \%$ efficiency to bring this to accretion disk scales (Müller-Sánchez et al. 2009; Schartmann et al. 2010), this mass alone is sufficient to fuel M81 for $10 \mathrm{Gyr}$, but it would supply NGC 1068 for only 1 Myr. In this context, an accretion rate similar to that for NGC 1068 is clearly very significant and may

\footnotetext{
${ }^{13}$ Throughout this paper we use units of erg s ${ }^{-1}$ for luminosities. $L_{\mathrm{AGN}}$ refers to the bolometric luminosity of an AGN. $L_{14-195}$ is the observed luminosity in the $14-195 \mathrm{keV}$ band. $L_{2-10}$ and $L_{2-10}^{\text {int }}$ are, respectively, the observed and absorption corrected (i.e., intrinsic) luminosities in the $2-10 \mathrm{keV}$ band. $L_{12} \mu \mathrm{m}$ refers to the monochromatic nuclear $12 \mu \mathrm{m}$ luminosity, measured at subarcsecond spatial resolution (for details see Asmus et al. 2014). We also adopt the relations $\log L_{\mathrm{AGN}}=1.12 \log L_{14-195}-4.23$ (Winter et al. 2012) and $\log L_{2-10}^{\text {int }}=1.06 \log L_{14-195}-3.08$ (Winter et al. 2009).
}

only be sustained via a relatively efficient inflow, perhaps driven by a coherent dynamical mechanism on scales of $0.1-1 \mathrm{kpc}$ where the gas reservoir is $10^{8}-10^{9} M_{\odot}$ (Sakamoto et al. 1999; Mazzalay et al. 2013). In contrast, low accretion rates comparable to that for M81 could be supplied either by a gas reservoir on small scales or by rather inefficient inflow from circumnuclear scales. Observationally, such an effect is implicit in the relation between star formation age and accretion rate presented by Davies et al. (2007); while AGNs with higher luminosities $\left(\sim 10^{45} \mathrm{erg} \mathrm{s}^{-1}\right)$ or Eddington ratios (0.1-1) may be associated with young post-starbursts, this is not the case for AGNs with luminosities two orders of magnitude lower. It could also help to explain the results of a survey of molecular gas (Haan et al. 2009; Garciá-Burillo 2011) which found that gravitational torques could drive gas inwards for not more than half of their sample. The role of AGN luminosity has been highlighted in the context of molecular outflows, in the sense that only very luminous AGNs drive massive outflows (Sturm et al. 2011; Veilleux et al. 2013).

Our premise for defining a new sample is that the $\mathrm{BH}$ accretion rate, traced by the AGN luminosity, is a key issue that needs to be considered in order to provide a context both for the mechanisms driving and regulating inflow and outflow as well as the derived flow rates. To address this issue, our sample is based on bright local AGNs in which the accretion rate is relatively high. We plan to analyse them in the context of matching inactive galaxies, for which the accretion rate is, by definition, orders of magnitude lower.

An obvious concern here is the timescale of AGN variability (Novak et al. 2011; Hickox et al. 2014) with respect to the timescale of the phenomenon (e.g., starburst, dynamical process, etc.) that is being assessed in the context of accretion onto the black hole. If an inactive sample is used naively as a control in a direct comparison to the active sample, the implicit assumption must be that any link to accretion is strong enough to persist even when averaged over the lifetime of the phenomenon being studied. However, Davies et al. (2014) argue and show that with a more judicious use of an inactive sample, one can make progress even if this assumption is not strictly met.

Building on the smaller sample of Hicks et al. (2013) and Davies et al. (2014), and also on the work summarized by Storchi-Bergmann $(2014,2015)$, we plan to address a few key questions in a statistically robust way.

1. Does star formation play a decisive role in either driving or hindering gas inflow to AGNs? This question focusses on the central $100 \mathrm{pc}$, assessing whether there has been recent star formation, and if so whether it is still on-going or has ceased.

2. What mechanisms are responsible for driving gas from the kiloparsec scale into the central tens of parsecs, and what triggers these? This includes dynamical processes in the central kiloparsec as well as the role of the larger scale host galaxy and the influence of the local environment.

3. Do luminous Seyferts always drive ionized and/or molecular outflows, and how do they interact with the interstellar medium? This addresses the ubiquity and efficacy of a variety of emission lines as tracers of outflows, as well as quantifying the outflow velocities, rates, and mass loading. 
These-and other-questions will be addressed in future papers using a combination of high spectral resolution data from 0.4-2.3 $\mu \mathrm{m}$ taken with XShooter (Vernet et al. 2011) and high spatial resolution integral field spectroscopy in the $\mathrm{H}$ and $\mathrm{K}$ bands taken with SINFONI (Eisenhauer et al. 2003; Bonnet et al. 2004), both on the VLT. At the time of writing, XShooter observations have been completed for 8 Seyferts, and 10 inactive galaxies, and the remainder of the targets are scheduled; SINFONI observations are completed or scheduled for the first half of the sample.

\subsection{Active Galaxy Sample}

Our first criterion for selecting a sample, and the only astrophysical one for the active sample, is to define a set of local AGNs in a well-characterized way so that the selection effects are (as far as possible) understood. The largest optical or infrared surveys that select AGNs are often incomplete for nearby galaxies, and also tend to use AGN tracers that are anisotropic or impose a bias against star formation (by using large apertures to measure features that can be produced by both AGNs and star formation). For example, [O III] $] 25007 \AA$, a typical optical line used in the selection of AGNs, is not isotropic: in comparison to [O IV $] \lambda 25.9 \mu \mathrm{m}$, which correlates well with $10-200 \mathrm{keV}$ emission, it is underluminous in some Sy 2 s-especially those that are Compton thick-by up to an order of magnitude compared to Sy 1s (Meléndez et al. 2008; Diamond-Stanic et al. 2009; Weaver et al. 2010). The same effect is seen in the $2-10 \mathrm{keV}$ emission when compared to the [O IV] line. However, high ionization lines may not be an ideal way to select AGNs since they are not always observed: Koss et al. (2013) detected $\mathrm{Ne} \mathrm{V}$ only in 2/3 of luminous infrared galaxies for which an AGN was confirmed by the Swift-BAT survey.

In contrast to emission line and infrared tracers, the very hard 14-195 keV band of the Swift-BAT survey measures direct emission from the AGNs rather than scattered or reprocessed emission, and is much less sensitive to obscuration in the lineof-sight than softer X-ray or optical wavelengths (selecting only against highly Compton thick AGNs). Indeed it is widely accepted as the least biased survey for AGNs with respect to host galaxy properties, and as such it has been well studied. There is a vast amount of ancillary data on the larger scale host galaxy properties (Winter et al. 2009, 2010; Koss et al. 2010, 2011; Meléndez et al. 2014; Mushotzky et al. 2014), as well as analysis of the bolometric corrections which enable one to make an estimate of the AGN luminosities (Vasudevan et al. 2010; Winter et al. 2012; Meléndez et al. 2014). Because it is an all-sky survey with roughly uniform sensitivity, we can select a complete, volume-limited sample of AGNs. Furthermore, the continuous nature of the survey means that more recent versions of the catalog (58 and 70 months, Baumgartner et al. 2013) average over variability during the last 5-6 years.

In addition to the single astrophysical criterion described above, we also impose two observational criteria to ensure homogeneous high resolution and observability. Our sample therefore consists of all 20 AGNs in the 58 months Swift-BAT catalog that meet the following three criteria: (i) $14-195 \mathrm{keV}$ luminosities $\log L_{14-195}>42.5$ (using redshift distance), (ii) redshift $z<0.01$ (corresponding to a distance of $\lesssim 40 \mathrm{Mpc}$ ), and (iii) observable from the VLT $\left(\delta<15^{\circ}\right)$ so that they tend to be in the southern sky. Note that the first two criteria are adjusted to intersect the flux limit of the 58 months catalog for
$90 \%$ of the sky, that is $1.46 \times 10^{-11} \mathrm{erg} \mathrm{s}^{-1} \mathrm{~cm}^{-2}$. The selection, and its completeness, are graphically represented in Figure 1.

\subsection{Matched Inactive Sample}

The second criterion for our selection is that there should be a properly matched sample of inactive galaxies, so that it is possible to discern which features (stellar age, inflow rates, etc.) are related specifically to the AGN activity. We describe the selection of the inactive sample below. Although it has no part in the analysis presented in this paper in Sections 4-5, it does play a key role in many of the analyses which we will present in future papers.

The characteristics on which the inactive sample matching is based are: host galaxy morphology (Hubble type), inclination (axis ratio) and H-band luminosity (as a proxy for stellar mass). For the purposes of target selection, we have derived the inclination from the axis ratio without any compensation for finite thickness. This is not expected to yield a significant bias when comparing two galaxies with similar morphological type, and the inclinations of the host galaxies will be assessed more carefully in future analyses. The relation between $\mathrm{H}$-band luminosity and stellar mass has been calibrated from a sample of similar galaxies for which the stellar mass has been derived using multi-band photometry (Koss et al. 2011). Figure 2 shows that the scatter in this relation is $0.2 \mathrm{dex}$.

We have quantified the matching criteria above using a $\chi^{2}$ metric with tolerances of \pm 1 for Hubble type, $\pm 15^{\circ}$ for inclination, and \pm 0.3 dex for $\mathrm{H}$-band luminosity. In addition we require that the presence/absence of a bar is matched if possible. Finally, also where possible, we have selected inactive galaxies with redshifts less than or equal to those of their active pairs. All inactive galaxies were selected from the RC3 catalog (de Vaucouleurs et al. 1991), rejecting any listed in the Véron-Cetty and Véron (2010) catalog of known AGNs. While some may still host very low luminosity AGNs, the decisive factor is that (based on the lack of any obvious signature of $\mathrm{BH}$ acretion in optical, radio, and X-ray data) it is orders of magnitude weaker than in the X-ray selected sample.

The inactive galaxy selection is based on matching the characteristics of individual galaxies in the active sample. This is used as a robust way to ensure that the samples, as a whole, match; it is not specifically our intention to compare pairs of active and inactive galaxies. Part of the reason is that some inactive galaxies are a good match for several active galaxies, which means there is no unique pair matching. The final matched inactive sample contains 19 galaxies, which are listed in Table 2 together with the host properties used for the selection process.

A graphical comparison of the main host galaxy properties is given in Figure 3. By design the distributions of these properties should be similar, as is apparent from the figure. This is confirmed for $L_{\mathrm{H}}$, axis ratio, and Hubble stage, by Kolmogorov-Smirnov (KS) tests which indicate that the differences are not significant. Formally, the probabilities that the differences between the active and inactive samples could arise by chance exceed $20 \%$. Both the active and inactive samples show the same distribution in H-band luminosity: the active sample has a mean of $\log L_{\mathrm{H}}\left[L_{\odot}\right]=10.3$ with a $1 \sigma$ spread of 0.3 while the inactive sample has a mean of 10.2 and a spread of 0.4 . Both samples cover the full range of axis ratio (or equivalently inclination), with about half the sample each side of 0.7 , equivalent to $45^{\circ}$ for a disk. And both samples 


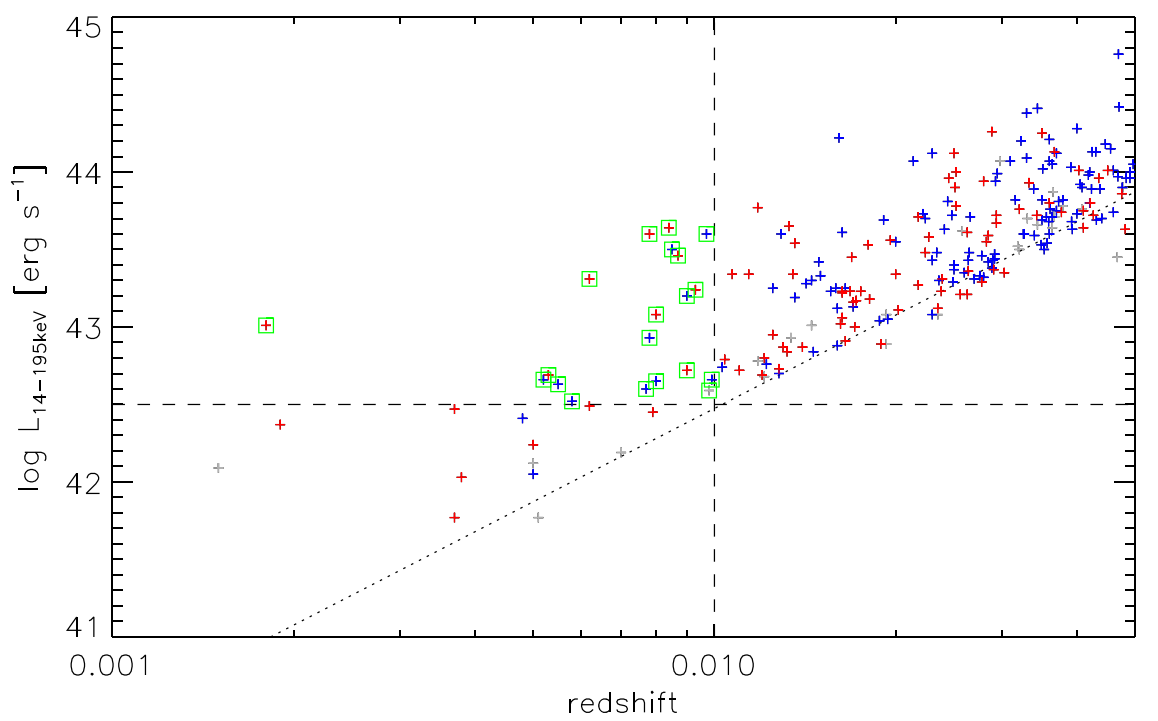

Figure 1. Plot of the redshift vs. luminosity for AGNs in the 58 months Swift-BAT survey that are readily observable from the VLT, i.e., with $\delta<15^{\circ}$, truncated at $z=0.05$. These have been color coded blue/red for Sy $1 /$ Sy 2 where such classifications are available (note that only "class 4 " AGNs are within our luminosity and redshift range). The dotted line is the flux limit for $90 \%$ of the sky. The dashed lines indicate the distance and luminosity thresholds for our selection in order to have a complete sample - for which the selected targets are marked with green. Luminosities in this plot are based on distances derived from the uncorrected redshift, as used in the initial target selection. These may differ slightly from the distances listed in Table 1 which are, where possible, redshift independent or corrected for peculiar velocities.

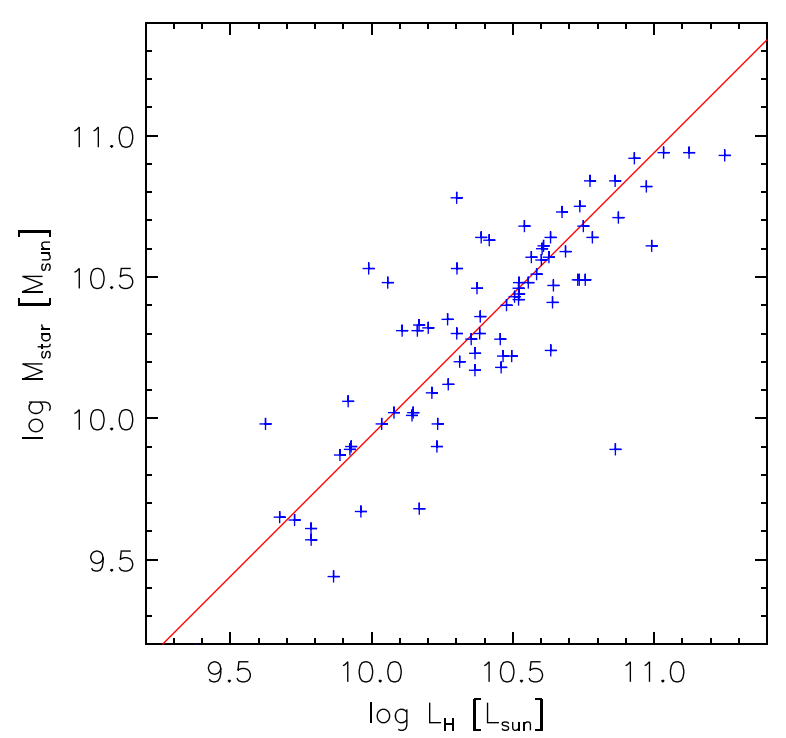

Figure 2. Comparison of the stellar mass $M_{\text {star, }}$, derived from multi-band photometry, to the integrated H-band luminosity $L_{\mathrm{H}}$ for a sample of AGNs and other galaxies similar to that presented here (Koss et al. 2011). There is a clear relation with a scatter of $\sim 0.2$ dex, showing that $L_{\mathrm{H}}$ can be used as a proxy for $M_{\text {star }}$.

cover a wide range of morphological types, with a clear preference for Hubble stage at $\sim 1$ corresponding to S0 and Sa "early disk" types. This distribution is consistent with the analysis presented by Koss et al. (2011), who found for a larger sample of Swift-BAT AGNs that only $\sim 10 \%$ were in ellipticals, while $\sim 30 \%$ had intermediate (S0) and $\sim 40 \%$ early type spiral $(\mathrm{Sa}-\mathrm{Sb})$ host galaxies. Koss et al. (2011) noted that these fractions differ from the distribution of normal galaxies-16\% ellipticals, $26 \%$ spirals. But the distribution of host types of the Swift-BAT AGNs are consistent with this once one takes into account the detection rate of Seyferts (rather than LINERs) reported by Ho (2008), which peaks for S0-Sb types. Davies et al. (2014) discussed the role of host type and environment in the context of the origin of the gas fuelling the AGN, and argued that this has an impact on what gas inflow mechanisms one may see in the circumnuclear region. This issue of morphology, including the presence or absence of a bar, will be revisited for our complete sample in a future work. The main point here is that the difference in distributions underlines the need to include morphology as a criterion for matching the inactive sample as we have done.

The largest difference between the samples occurs for the distance distribution, which a KS test indicates may be marginally significant at a level of $2.5 \sigma$. This is a direct result of our requirement to select, whenever possible, inactive galaxies that are closer, rather than more distant, to their active pair. We note that the active and inactive samples have mean distances of 31 and $24 \mathrm{Mpc}$, respectively, and in both cases the $1 \sigma$ spread is about $9 \mathrm{Mpc}$.

\section{AGN PROPERTIES}

In this section we compare various properties of the Sy $1 \mathrm{~s}$ and Sy $2 \mathrm{~s}$ in the active sample, as listed in Table 1, focussing on the absorbing column and its impact. We will, as our data become available, update the classifications of the AGNs if necessary. Similarly, and as applicable to the specific analyses we will perform, we will re-assess the host galaxy properties of the active and inactive samples: morphological classifications, whether they are barred, and their inclinations.

The sample was selected purely on $L_{14-195}$. Adopting a simple conversion to estimate the bolometric AGN luminosity as (Winter et al. 2012)

$$
\log L_{\mathrm{AGN}}=1.12 \log L_{14-195}-4.23
$$

(i.e., bolometric corrections of 5-10 for the luminosity range here) and without applying any absorption correction (see below), our complete volume limited sample has a median 
Table 1

Summary of AGN and Host Galaxy Properties

\begin{tabular}{|c|c|c|c|c|c|c|c|c|c|c|}
\hline $\begin{array}{l}\text { Name } \\
\text { (1) }\end{array}$ & $\begin{array}{c}\text { Distance } \\
(\mathrm{Mpc}) \\
(2)\end{array}$ & $\begin{array}{c}\text { AGN } \\
\text { Classification } \\
\text { (3) }\end{array}$ & $\begin{array}{c}\log L_{\mathrm{H}} \\
L_{\odot} \\
(4)\end{array}$ & $\begin{array}{c}\log L_{14-195} \\
\left(\mathrm{erg} \mathrm{s}^{-1}\right) \\
(5)\end{array}$ & $\begin{array}{c}\log L_{2-10} \\
\left(\mathrm{erg} \mathrm{s}^{-1}\right) \\
(6)\end{array}$ & $\begin{array}{c}\log L_{12 \mu \mathrm{m}} \\
\left(\mathrm{erg} \mathrm{s}^{-1}\right) \\
(7)\end{array}$ & $\begin{array}{c}\log N_{\mathrm{H}} \\
\left(\mathrm{cm}^{-2}\right) \\
(8)\end{array}$ & $\begin{array}{l}\text { Hubble } \\
\text { Stage } \\
(9)\end{array}$ & $\begin{array}{c}\text { Axis } \\
\text { Ratio } \\
(10)\end{array}$ & $\begin{array}{l}\text { Bar } \\
\text { (11) }\end{array}$ \\
\hline NGC 1365 & 18 & Sy $1.8^{\mathrm{a}}$ & 10.58 & 42.39 & 41.83 & 42.54 & $22.2^{\mathrm{d}}$ & 3 & 0.55 & $\mathrm{~B}$ \\
\hline MCG-05-14-012 & 41 & Sy $1.0^{\mathrm{a}}$ & 9.60 & 42.60 & 41.63 & $\cdots$ & $\leqslant 21.9$ & -1 & 0.86 & $\cdots$ \\
\hline NGC 2110 & 34 & Sy $2(1 \mathrm{~h})^{\mathrm{a}}$ & 10.44 & 43.64 & 42.53 & 43.04 & $23.0^{\mathrm{e}}$ & -3 & 0.74 & $\mathrm{AB}$ \\
\hline NGC 2992 & 36 & Sy $1.8^{\mathrm{a}}$ & 10.31 & 42.62 & 42.05 & 42.87 & 21.7 & 1 & 0.30 & $\ldots$ \\
\hline MCG-05-23-016 & 35 & Sy $1.9^{\mathrm{a}}$ & 9.94 & 43.47 & 43.11 & 43.42 & 22.2 & -1 & 0.45 & $\ldots$ \\
\hline NGC 3081 & 34 & Sy $2(1 \mathrm{~h})^{\mathrm{a}}$ & 10.15 & 43.06 & 41.54 & 42.75 & 23.9 & 0 & 0.77 & $\mathrm{AB}$ \\
\hline NGC 3783 & 38 & Sy $1.2^{\mathrm{a}}$ & 10.29 & 43.49 & 43.12 & 43.47 & 20.5 & 1.5 & 0.89 & B \\
\hline NGC 4235 & 37 & Sy 1.2 & 10.43 & 42.72 & 41.66 & 42.17 & 21.3 & 1 & 0.22 & $\ldots$ \\
\hline NGC 4388 & 39 & Sy 2 (1h) & 10.65 & 43.70 & 42.57 & 42.93 & $23.5^{\mathrm{d}}$ & 3 & 0.18 & B \\
\hline NGC 4593 & 37 & Sy $1.0-1.2^{\mathrm{a}}$ & 10.59 & 43.16 & 42.77 & 42.97 & $\leqslant 19.2$ & 3 & 0.74 & B \\
\hline NGC 5128 (Cen A) & 3.8 & Sy 2 & 10.22 & 42.38 & 41.50 & 41.82 & $23.1^{\mathrm{d}}$ & -2 & 0.78 & $\cdots$ \\
\hline ESO 021-G004 & 39 & $\mathrm{Sy}^{\mathrm{b}}$ & 10.53 & 42.49 & 41.21 & $\ldots$ & 23.8 & -0.4 & 0.45 & $\cdots$ \\
\hline MCG-06-30-015 & 27 & Sy 1.2 & 9.59 & 42.74 & 42.51 & 42.87 & 20.9 & -5 & 0.60 & $\cdots$ \\
\hline NGC 5506 & 27 & Sy 2 (1i) & 10.09 & 43.32 & 42.91 & 43.28 & 22.4 & 1 & 0.23 & $\cdots$ \\
\hline NGC 5728 & 39 & Sy 2 & 10.56 & 43.21 & 41.41 & 42.35 & 24.2 & 1 & 0.57 & $\mathrm{~B}$ \\
\hline ESO 137-G034 & 35 & Sy 2 & 10.44 & 42.62 & 40.86 & $\ldots$ & 24.3 & 0 & 0.79 & $\mathrm{AB}$ \\
\hline NGC 6814 & 23 & Sy 1.5 & 10.31 & 42.69 & 42.17 & 42.18 & 21.0 & 4 & 0.93 & $\mathrm{AB}$ \\
\hline NGC 7172 & 37 & Sy $2(1 \mathrm{i})^{\mathrm{c}}$ & 10.43 & 43.45 & 42.53 & 42.88 & 22.9 & 1.4 & 0.56 & $\ldots$ \\
\hline NGC 7213 & 25 & Sy 1 & 10.62 & 42.50 & 41.95 & 42.58 & $\leqslant 20.4$ & 1 & 0.90 & $\ldots$ \\
\hline NGC 7582 & 22 & Sy $2(1 \mathrm{i})^{\mathrm{c}}$ & 10.38 & 42.67 & 41.12 & 42.81 & $24.2^{\mathrm{a}}$ & 2 & 0.42 & B \\
\hline
\end{tabular}

Note. Columns: (1) common name; (2) distances are taken from NED, using redshift independent estimates or peculiar velocity corrections derived by Theureau et al. (2007) where possible (except for NGC 5128 where the distance is from Harris et al. 2010), and so may differ slightly from the distance based on the uncorrected redshift used in the initial selection; (3) AGN type as given in NED, except where indicated for the eight AGNs that we can independently classify, with additional information: 1i-near-infrared broad lines, $1 \mathrm{~h}$ - polarized broad line emission; (4) integrated H-band luminosity (given by the 2MASS total magnitude; Skrutskie et al. 2006) as a proxy for stellar mass; (5) observed 14-195 keV luminosity (70 months average) from Swift-BAT (Baumgartner et al. 2013); (6) observed 2-10 keV luminosity (single epoch) from C. Ricci et al. (2015, in preparation); (7) nuclear $12 \mu \mathrm{m}$ luminosity from Asmus et al. (2014) (MCG-05-14-012, ESO 021-G004, and ESO 137-G034 are not included in this catalog and, as far as we know, do not have $12 \mu \mathrm{m}$ measurements on arcsec scales); (8) neutral absorbing column, from C. Ricci et al. (2015, in preparation) based on modeling 0.3-150 keV spectrum; (9) Hubble stage (NED); (10) axis ratio (NED); (11) B and AB denote presence of a bar or weak bar respectively (NED).

${ }^{a}$ Confirmed or updated based on our available XSHOOTER data (A. Schnorr-Müller et al. 2015, in preparation). Note that NGC 4593 is ambiguous because it is close to the boundary between Sy 1 and Sy 1.2; MCG-05-23-016 has weak broad H $\alpha$; NGC 2110 is changed from "Sy 2 (1i)" to a simple "Sy 2," because the polarized broad line has a double peaked profile which is not seen at near-infrared wavelengths.

${ }^{\mathrm{b}}$ ESO 021-G004 has no optical classification, and our XSHOOTER data for this object have not yet been taken.

${ }^{c}$ We have added a "1i" qualifier to the classifications of NGC 7172 because Smajić et al. (2012) show evidence for broad Pa $\alpha$ and Br $\gamma$, and NGC 7582 because Reunanen et al. (2003) find broad $\operatorname{Br} \gamma$.

${ }^{\mathrm{d}}$ Variations in $N_{\mathrm{H}}$ between Compton thick and thin have been reported for NGC 1365 (Risaliti et al. 2009) and NGC 7582 (Bianchi et al. 2009 ). Compton thin variations in $N_{\mathrm{H}}$ have been reported for NGC 4388 (Elvis et al. 2004) and NGC 5128 (Beckmann et al. 2011).

e The absorbing column for NGC 2110 includes partial covering absorbers, and the value given here is weighted by covering factor. Details are given in C. Ricci et al. (2015, in preparation); see also Evans et al. (2007).

luminosity of $\log L_{\mathrm{AGN}}=43.5$ with a $1 \sigma$ distribution of 0.5 dex.

Table 1 shows that our selection has yielded similar numbers of Sy 1s and Sy 2s, and the first panel of Figure 4 indicates that any difference in their $L_{14-195}$ distributions is not significant. This suggests that, for the number of objects in the sample, any preference to select either Sy 1s or Sy $2 \mathrm{~s}$ is not significant. For both $L_{14-95}$ and $L_{12 \mu \mathrm{m}}$, a KS test indicates that the probability of the difference between the Sy 1 and Sy 2 distributions arising by chance exceeds $45 \%$. The difference for the observed $L_{2-10}$ is also not enough to be significant. It does, however, have a measurable impact on the ratio of the observed $14-195 \mathrm{keV}$ and 2-10 keV luminosities, which is 4 for the Sy 1 s and 17 for the Sy 2s. The same characteristic is clearly seen in the left panel of Figure 5 where $L_{14-195}$ is plotted versus observed $L_{2-10}$. Here $S y 1 \mathrm{~s} / 2 \mathrm{~s}$ are represented by blue/red color, respectively. The Sy 1s follow the relation between
$L_{14-195}$ and $L_{2-10}^{\text {int }}$ derived by Winter et al. (2009)

$$
\log L_{2-10}^{\mathrm{int}}=1.06 \log L_{14-195}-3.08,
$$

(in the luminosity range here, this relation is approximately equivalent to $\left.\log L_{2-10}^{\text {int }} \sim \log L_{14-195}-0.5\right)$ while many of the Sy 2 s do not. The reason is due to their different absorbing columns $N_{\mathrm{H}}$, which is denoted by open/filled symbols in Figure 5. And indeed, the observed $L_{14-195} / L_{2-10}$ ratio can in principle be used to give an estimate of $N_{\mathrm{H}}$ (Koss et al. 2013).

The difference in $N_{\mathrm{H}}$ is clearly seen in the center right panel of Figure 4, and a KS test gives it a significance of $3.7 \sigma$. Values for $N_{\mathrm{H}}$ are taken from C. Ricci et al. (2015, in preparation), who derived absorbing columns for many of the Swift-BAT AGNs in a consistent way, fitting the $0.3-150 \mathrm{keV}$ spectral energy distribution. The basic model includes an absorbed cutoff power-law continuum plus a reflection component (for details see C. Ricci et al. 2015, in preparation). For sources with $N_{\mathrm{H}} \lesssim 10^{22} \mathrm{~cm}^{-2}$, 
Table 2

Summary of Control Sample Properties

\begin{tabular}{lccccc}
\hline \hline Name & $\begin{array}{c}\text { Distance } \\
(\mathrm{Mpc})\end{array}$ & $\begin{array}{c}\log L_{\mathrm{H}} \\
L_{\odot}\end{array}$ & $\begin{array}{c}\text { Hubble } \\
\text { Stage }\end{array}$ & $\begin{array}{c}\text { Axis } \\
\text { Ratio }\end{array}$ & Bar \\
$(1)$ & $(2)$ & $(3)$ & $(4)$ & $(5)$ & $(6)$ \\
\hline NGC 718 & 23 & 9.89 & 1 & 0.87 & $\mathrm{AB}$ \\
NGC 1079 & 19 & 9.91 & 0 & 0.60 & $\mathrm{AB}$ \\
NGC 1315 & 21 & 9.47 & -1 & 0.89 & $\mathrm{~B}$ \\
NGC 1947 & 19 & 10.07 & -3 & 0.87 & $\ldots$ \\
ESO 208-G021 & 17 & 10.88 & -3 & 0.70 & $\mathrm{AB}$ \\
NGC 2775 & 21 & 10.45 & 2 & 0.77 & $\ldots$ \\
NGC 3175 & 14 & 9.84 & 1 & 0.26 & $\mathrm{AB}$ \\
NGC 3351 & 11 & 10.07 & 3 & 0.93 & $\mathrm{~B}$ \\
ESO 093-G003 & 22 & 9.86 & 0.3 & 0.60 & $\mathrm{AB}$ \\
NGC 3717 & 24 & 10.39 & 3 & 0.18 & $\ldots$ \\
NGC 3749 & 42 & 10.40 & 1 & 0.25 & $\ldots$ \\
NGC 4224 & 41 & 10.48 & 1 & 0.35 & $\ldots$ \\
NGC 4254 & 15 & 10.22 & 5 & 0.87 & $\ldots$ \\
NGC 4260 & 31 & 10.25 & 1 & 0.31 & $\mathrm{~B}$ \\
NGC 5037 & 35 & 10.30 & 1 & 0.32 & $\ldots$ \\
NGC 5845 & 25 & 10.46 & -4.6 & 0.63 & $\ldots$ \\
NGC 5921 & 21 & 10.08 & 4 & 0.82 & $\mathrm{~B}$ \\
IC 4653 & 26 & 9.48 & -0.5 & 0.63 & $\mathrm{~B}$ \\
NGC 7727 & 26 & 10.41 & 1 & 0.74 & $\mathrm{AB}$ \\
\hline
\end{tabular}

Columns: (1) common name; (2) distances are, where possible, redshift independent (NED); (3) integrated H-band luminosity (as a proxy for stellar mass). (4) Hubble stage (NED); (5) axis ratio (NED); (6) B and AB denote presence of a bar or weak bar respectively (NED).

they included various additional components if statistically needed: a blackbody (for the soft excess), partially covering ionized absorption, a cross-calibration constant (for possible variability between the non-simultaneous soft X-ray and Swift$B A T$ observations), and an iron line (or other emission lines in that region). Similarly, for the more obscured sources, some additional components were added if required: a scattered component, a collisional plasma, emission lines, and a crosscalibration constant. Note that in the column densities given in Table 1 we do not include warm absorbers, which arise in ionized out-flowing gas and are observed in about $50 \%$ of Sy $1 \mathrm{~s}$ (Komossa 1999; Blustin et al. 2005; Winter et al. 2012). The difference between the neutral absorption in Sy $1 \mathrm{~s}$ and $2 \mathrm{~s}$ is significant. The mean for the Sy $1 \mathrm{~s}$ is $\log N_{\mathrm{H}}\left[\mathrm{cm}^{-2}\right] \sim 21.1$ while for Sy $2 \mathrm{~s}$ it is $\log N_{\mathrm{H}}\left[\mathrm{cm}^{-2}\right]=23.5$.

Models of the propagation of X-rays through gas in various geometries around a central source (Matt et al. 1999; Brightman \& Nandra 2011; Yaqoob 2012; C. Ricci et al. 2015, in preparation) indicate that $N_{\mathrm{H}}$ can account for the different luminosity ratio between the Sy 1 s and Sy 2 s. These models indicate that the flux correction for the $14-195 \mathrm{keV}$ band is negligible up to $10^{23} \mathrm{~cm}^{-2}$, and reaches a factor 2 around $(2-3) \times 10^{24} \mathrm{~cm}^{-2}$, and a factor 10 at $10^{25} \mathrm{~cm}^{-2}$ (whether higher corrections for larger columns are required depends on the reflection component). As such, even the absorption corrected 14-195 keV luminosities do not, based on a KS test, yield a significant difference between Sy 1s and Sy 2s. On the other hand, large flux corrections, greater than a factor 2, to $L_{2-10}$ are already required for absorbing columns of $\sim 10^{23} \mathrm{~cm}^{-2}$, increasing to a factor 10 at $\sim 5 \times 10^{23} \mathrm{~cm}^{-2}$. If not accounted for, this can lead to a bias when selecting via the $2-10 \mathrm{keV}$ band, since the impact on the observed flux means that some Sy 2s may be excluded from a sample even before such a correction can be made.
This effect is much reduced when selecting in the $14-195 \mathrm{keV}$ band. Its impact is apparent in the distribution of $N_{\mathrm{H}}$ for the Sy 2s: as expected from Risaliti et al. (1999), most of the sources have $N_{\mathrm{H}} \gtrsim 10^{23} \mathrm{~cm}^{-2}$. And (allowing for variations in $N_{\mathrm{H}}$ ) 3-5 of the sources have $N_{\mathrm{H}}>10^{24} \mathrm{~cm}^{-2}$, consistent with the expectation that $10 \%-30 \%$ of AGNs are Compton thick (Treister et al. 2009; Alexander et al. 2013). But in contrast to Risaliti et al. (1999) there are none with $N_{\mathrm{H}} \gtrsim 10^{25} \mathrm{~cm}^{-2}$, which is simply due to the absorption in the band at such high columns. However, we expect very few local sources to fall in this regime. NGC 1068 is the best known example of one that does, and hence is excluded from the sample: although its $L_{14-195} / L_{2-10} \sim 10$ is not immediately indicative of a high column, early estimates (Matt et al. 1997) suggested it is highly Compton thick, and fits to recent hard $\mathrm{X}$-ray data require several components with columns up to $10^{25} \mathrm{~cm}^{-2}$ (Bauer et al. 2014).

The final comparison is of the nuclear $12 \mu \mathrm{m}$ luminosities, taken from Asmus et al. (2014). These data were observed at subarcsecond resolution, and so resolve out mid-infrared continuum due to star formation in the circumnuclear region. Gandhi et al. (2010) and Asmus et al. (2012) have shown that there is a very good correlation between $L_{2-10}^{\text {int }}$ and $L_{12 \mu \mathrm{m}}$, with no major difference between Sy 1s and Sy 2 s. Our comparison in Figures 4 and 5 confirms that this is also the case for our sample and that there is little dependence on $L_{12 \mu \mathrm{m}}$ or its relation to $L_{14-195}$ with either AGN type or $N_{\mathrm{H}}$. Note, however, Burtscher et al. (2015) point out that the nuclear $12 \mu \mathrm{m}$ continuum does show some signs of a slight anisotropy, which can also be seen in the right panel of Figure 5.

\section{OPTICAL OBSCURATION AT LOW LUMINOSITY}

The proportion of AGNs with substantial optical obscuration and/or X-ray absorption, and their dependence on luminosity, offers insight into the properties of the obscuring structures around the AGN. In this section we focus on low luminosities, in the range $42.5<\log L_{14-195}<44$. After clarifying in Section 4.1 the definitions we use for optically obscured and X-ray absorbed AGNs, and summarizing the various types of AGNs that are found, we discuss X-ray unabsorbed Sy $2 \mathrm{~s}$ in Section 4.2, and put this in the context of the Swift-BAT sample in Section 4.3 to assess how rare such objects really are.

\subsection{Definitions and Classifications}

Much of the discussion below is set in the context of Merloni et al. (2014). As such, we follow these authors and adopt $N_{\mathrm{H}}>10^{21.5} \mathrm{~cm}^{-2}$ as the criterion for defining an AGN to be $\mathrm{X}$-ray absorbed. For typical gas-to-dust ratios this is equivalent to an optical screen extinction of $A_{V} \sim 2 \mathrm{mag}$, sufficient to mildy obscure the line of sight to the BLR at visible wavelengths. ${ }^{14}$ In our volume limited $14-195 \mathrm{keV}$ sample, we find $50 \%-60 \%$ of the AGNs are optically obscured while $\sim 60 \%$ are X-ray absorbed. Most of the obscured AGNs are both optically obscured and X-ray absorbed; none are just

\footnotetext{
${ }^{14}$ We do not distinguish a category of obscuration in which the broad emission lines are obscured at optical wavelengths but can be detected at nearinfrared wavelengths. These objects, which are sometimes classified as Sy 1i, are considered to be Sy $2 \mathrm{~s}$ in this work. We note, however, that like Sy $1.8-1.9 \mathrm{~s}$ they are close to the boundary of optically obscured/unobscured and X-ray absorbed/unabsorbed. And there are good reasons to consider them instead as Sy 1 as discussed by Burtscher et al. (2015).
} 

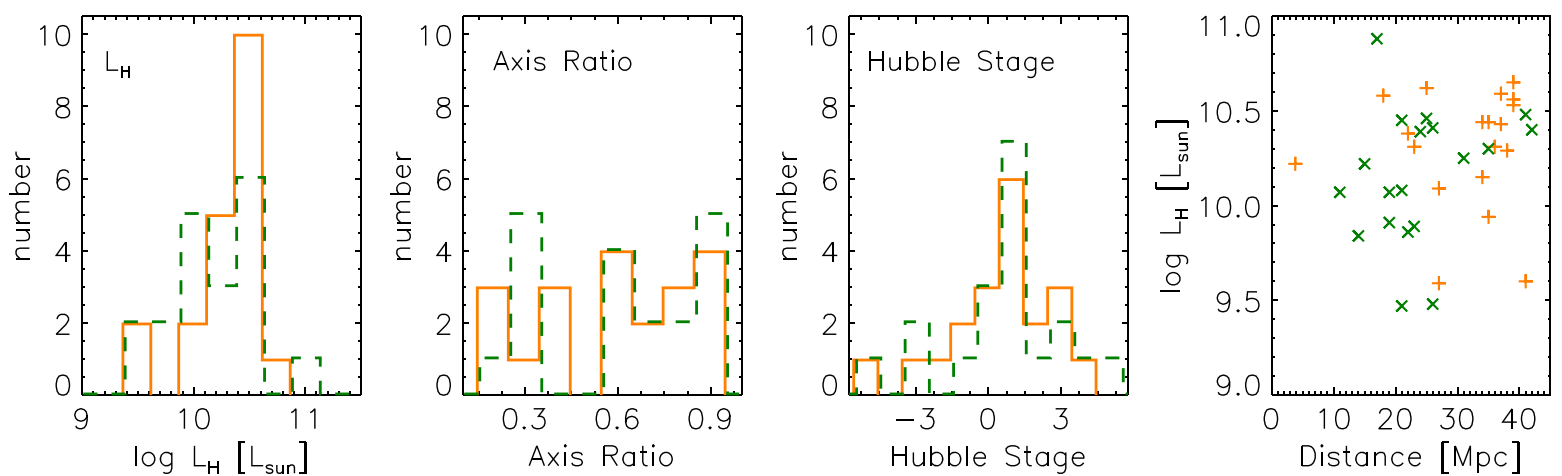

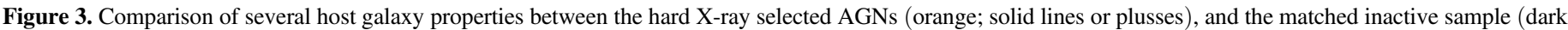

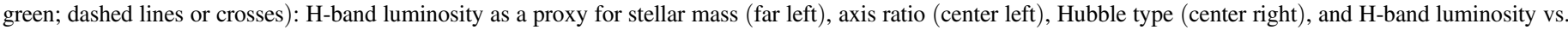

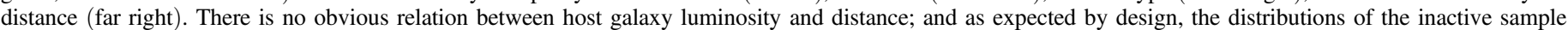

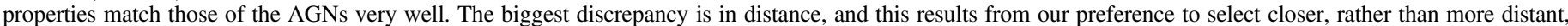
inactive galaxies when possible.

optically obscured; only three are just X-ray absorbed. These exceptions are "minor" since they are all close to the boundary between the regimes. The X-ray absorbed AGN NGC 1365 is optically classified as Sy 1.8 and, although in many studies is taken to be a Sy 2, here we adopt a strict definition and so classify it as Sy 1. Similarly MCG-05-23-016 is X-ray absorbed and has only weak broad $\mathrm{H} \alpha$, hence its previous classification as a Sy 2 which we have revised to Sy 1.9. The Sy 1.8 NGC 2992 has a column only marginally above our X-ray absorption threshold and so is on the borderline in both classifications. It would be classified as X-ray unabsorbed according to other definitions that set the threshold at $10^{22} \mathrm{~cm}^{-2}$ (Panessa \& Bassani 2002). As such, there could be two more optically obscured and one less X-ray absorbed AGN than found using the strict definitions we apply in Figure 6. This is reflected by the ranges shown in Figure 7.

The joint optical/X-ray classifications are summarized in Figure 6 where we adopt the terminology of Merloni et al. (2014) in which the first/second digit of the classification denotes the optical/X-ray type. This leads to the following types:

type 11: optically unobscured and X-ray unabsorbed; type 22: optically classified as Sy 2 and X-ray absorbed; type 21: optically classified as Sy 2 but X-ray unabsorbed; type 12: optically unobscured but X-ray absorbed.

Thus, with reference to the curves in Figure 7 adapted from Merloni et al. (2014) to the $14-195 \mathrm{keV}$ luminosity scale, we also have:

type 22+12: all X-ray absorbed AGNs (regardless of their optical classification);

type 22+21: all AGNs optically classified as Sy 2 (independent of whether they are X-ray absorbed).

Comparing the AGNs in our sample to the left edge of Figure 7 immediately highlights a discrepancy. Above, we noted that $50 \%-60 \%$ of our complete sample are optically obscured and classed as Sy 2s, a fraction similar to that estimated by Lawrence \& Elvis (2010). However, Merloni et al. (2014) class $\sim 90 \%$ of their AGNs in an overlapping luminosity range $\log L_{14-195} \sim 43-43.5$ as Sy 2 s. The difference is due to the large fraction of AGNs classified by these authors as type 21 , that is X-ray unabsorbed Sy 2 galaxies
(Pappa et al. 2001; Panessa \& Bassani 2002; Brightman \& Nandra 2008; Bianchi et al. 2012).

\subsection{How Common are X-Ray Unabsorbed Seyfert $2 s$ ?}

X-ray unabsorbed Sy 2s are believed to be galaxies with a direct view to the AGNs but in which there is no BLR (such objects are also known as pure or true Sy $2 \mathrm{~s}$ ). This view is supported by, for example, the six objects identified by Hawkins (2004) as having optical spectra typical of Sy $2 \mathrm{~s}$ $\left(\mathrm{H} \beta \mathrm{FWHM}<1000 \mathrm{~km} \mathrm{~s}^{-1}\right.$ and $\left.\left[\mathrm{O}_{\mathrm{III}}\right] / \mathrm{H} \beta>3\right)$ but large amplitude variations typical of Sy $1 \mathrm{~s}$. Subsequent X-ray observations of three by Gliozzi et al. (2007) confirmed that these do not have significant absorption in the $0.3-8 \mathrm{keV}$ band. These authors also showed that these AGNs are relatively luminous (intrinsic $\log L_{0.5-8}>43.2$ ). Thus, despite their rather low Eddington ratios $L_{\text {bol }} / L_{\text {Edd }}<0.01$ (Gliozzi et al. 2007), the absence of a BLR cannot be explained by low luminosities and/or accretion rates either being unable to sustain a disk wind (Nicastro 2000; Elitzur \& Ho 2009) or meaning that high dispersion prevents BLR clouds from surviving (Laor 2003). At present, the reason that they may not have a BLR is still open.

These objects may be related to the Sy $2 \mathrm{~s}$ for which spectropolarimetry shows no evidence of a hidden BLR. There have been many studies of polarized emission from hidden BLRs. The largest indicate that Sy $2 \mathrm{~s}$ in which a hidden BLR has not been detected have less luminous AGNs than other Sy 2 s but are not more optically obscured (Tran 2001, 2003; Gu \& Huang 2002)—qualitatively, but not necessarily quantitatively, consistent with the idea that BLR may not form in low luminosity AGNs. It also suggests that a BLR is completely absent in at least $50 \%$ of Sy 2 s. However, this fraction is difficult to put in perspective because of complications due to the impact of detection limits as well as the expected scattering efficiency. The latter issue was addressed by Heisler et al. (1997) who showed that the ability to detect polarized emission was related to the far-infrared colors in a way that suggested the scattering particles were in the throat of the torus, and so even they would be hidden at high inclinations. Following on from work of Ramos Almeida et al. (2011) which shows that the intrinsic properties (rather than just the inclination) of the tori in Sy $1 \mathrm{~s}$ and Sy $2 \mathrm{~s}$ are different, Ichikawa et al. (2015) have fitted torus models to Sy $2 \mathrm{~s}$ with and without polarized emission. Their results again 

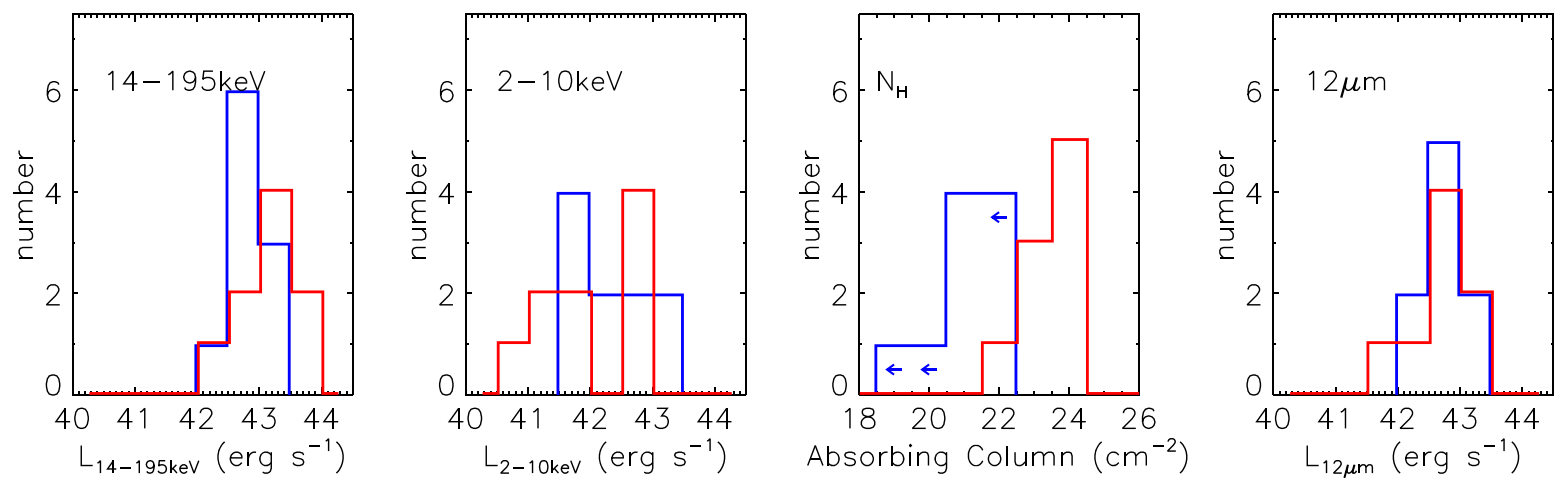

Figure 4. Comparison of nuclear properties of Sy 1s (blue) and Sy 2s (red). There are no significant differences between Sy $1 \mathrm{~s}$ and Sy 2 s for any of the luminosity distributions shown. This holds even for the absorption corrected 14-195 keV luminosity (not shown), despite the tantalizing hint of a difference. In contrast, the difference in $\log N_{\mathrm{H}}$ is $3.7 \sigma$. Far left: the sample was selected according to $14-195 \mathrm{keV}$ luminosity, and shows no significant bias toward either Sy $1 \mathrm{~s}$ or Sy $2 \mathrm{~s}$. Center left: given the small numbers of objects, there is also no significant difference in the observed 2-10 keV luminosities. Center right: in contrast, and as expected, Sy $2 \mathrm{~s}$ do exhibit higher absorbing columns, with a median of $>10^{23} \mathrm{~cm}^{-2}$ in comparison to $\sim 10^{21} \mathrm{~cm}^{-2}$ for the Sy $1 \mathrm{~s}$ (note we have marked AGNs with no measurable neutral absorption as limits). Far right: the nuclear mid-infrared luminosity shows a remarkably narrow distribution, similar to that of the 14-195 keV luminosity, with no clear difference between Sy 1s and Sy 2s.
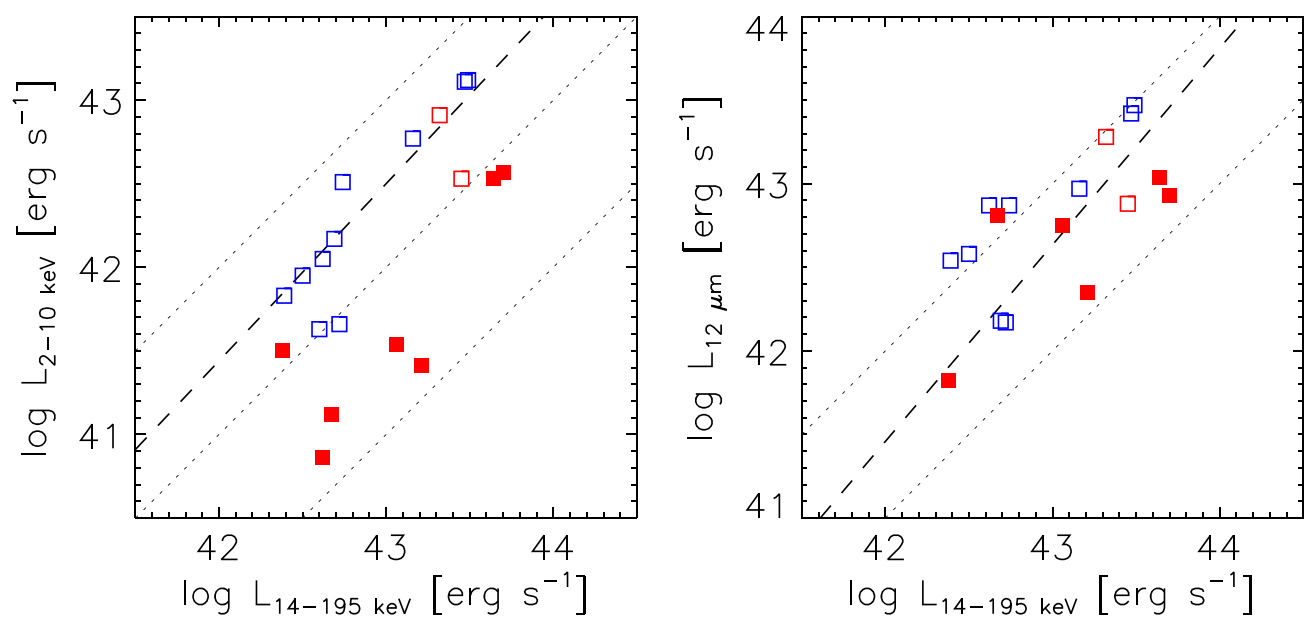

Figure 5. Comparison of nuclear X-ray and mid-infrared luminosities of Sy $1 \mathrm{~s}$ and Sy 2s (denoted by blue and red color respectively). Absorbing column is represented by open/filled symbols: filled squares have $N_{\mathrm{H}} \geqslant 10^{23} \mathrm{~cm}^{-2}$ while open squares have $N_{\mathrm{H}}<10^{23} \mathrm{~cm}^{-2}$ (as discussed in Section 3, this threshold is where $N_{\mathrm{H}}$ starts to have an impact on the $2-10 \mathrm{keV}$ band). The two red open squares are both AGNs classified as Sy 2(1i). Burtscher et al. (2015) argue that, based on their physical properties, these should be considered more like Sy 1 than Sy 2. Left: $L_{14-195}$ vs. observed $L_{2-10}$. From top left to bottom right, the dotted lines trace ratios of $L_{14-195} / L_{2-10}=1,10,100$ (indicative of increasing absorbing column, see Koss et al. 2013). The relation of Winter et al. (2009) for $L_{2-10}^{\text {int }}$ is indicated by the dashed line. Sy $1 \mathrm{~s}$ and other AGNs with low $N_{\mathrm{H}}$ follow this relation, indicating that for these $L_{2-10} \sim L_{2-10}^{\text {int }}$. Right: $L_{14-195}$ vs. nuclear $L_{12} \mu \mathrm{m}$. From top left to bottom right, the dotted lines indicate ratios of $L_{14-195} / L_{12 \mu m}=1,10$. The dashed line denotes the relation of Gandhi et al. (2010), replacing $L_{2-10}^{\text {int }}$ with $L_{14-195}$ according to Winter et al. (2009). To first order, the data follow this relation without any dependency on $N_{\mathrm{H}}$ or AGN type.

indicate that whether a hidden BLR is seen depends on the location of the scattering material in the throat of the torus; but that rather than inclination, it is the opening angle and covering factor of the torus that determines this difference. As such, the fraction of Sy $2 \mathrm{~s}$ in which polarized scattered BLR emission is not observed could be very different from the fraction without a hidden BLR. Since, based on these models, the polarized BLR emission may in some cases be only relatively lightly obscured, deep and/or near-infrared spectropolarimetry may shed further light on this issue. NGC 7172 and NGC 7582 (see Table 1) are cases in point here: Bian \& Gu (2007) and Marinucci et al. (2012) both report that no polarized broad $\mathrm{H} \alpha$ has been detected but Smajić et al. (2012) have seen broad Pa $\alpha$ and $\mathrm{Br} \gamma$ directly in NGC 7172 and Reunanen et al. (2003) broad $\mathrm{Br} \gamma$ in NGC 7582.

Despite the uncertainties, the evidence that at least some relatively luminous AGNs do not have a BLR is convincing. The key question for this paper is how common such objects are, since this can have a significant impact on the total fraction of AGNs optically classified as Sy 2.

In their analysis, Risaliti et al. (1999) found that about $4 \%$ of Sy 2 galaxies had $N_{\mathrm{H}}<10^{22} \mathrm{~cm}^{-2}$. However, Panessa \& Bassani (2002) presented a sample of 17 objects which had been classified as Sy 2 in the literature, and which had similarly low column densities. They suggested that X-ray unabsorbed Sy 2s may be much more common, being $10 \%-30 \%$ of all Sy $2 \mathrm{~s}$. More recently, other authors have confirmed that such objects do exist, but without a consensus on how common they are, because many candidates are ruled out on closer examination. For example, Brightman \& Nandra (2008) found that of eight candidates, four were underluminous in the $2-10 \mathrm{keV}$ bands suggesting that they were Compton thickwith the unabsorbed softer X-rays originating in a scattered continuum or from host galaxy contamination. Similarly, in an examination of eight candidates-including some from the Panessa \& Bassani (2002) sample_Bianchi et al. (2012) ruled 


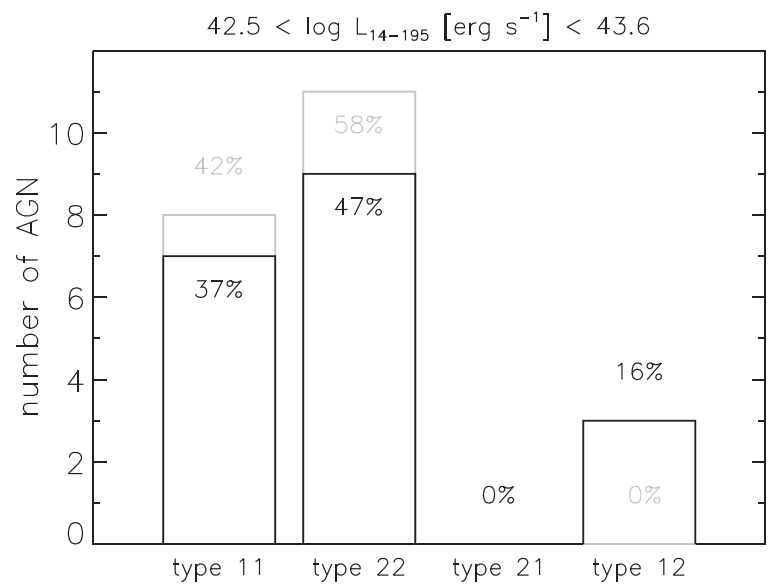

Figure 6. Fractions of optically obscured and X-ray absorbed AGNs in our complete volume limited Swift-BAT sample (see also Figure 7). The first/ second digit of the type codes for optical/X-ray obscuration as described in Section 4.1. The black lines denote the strict definitions. The gray lines show the impact of relaxing the definitions slightly: the three type 12 AGNs would be reclassified as one type 11 and two type 22 .

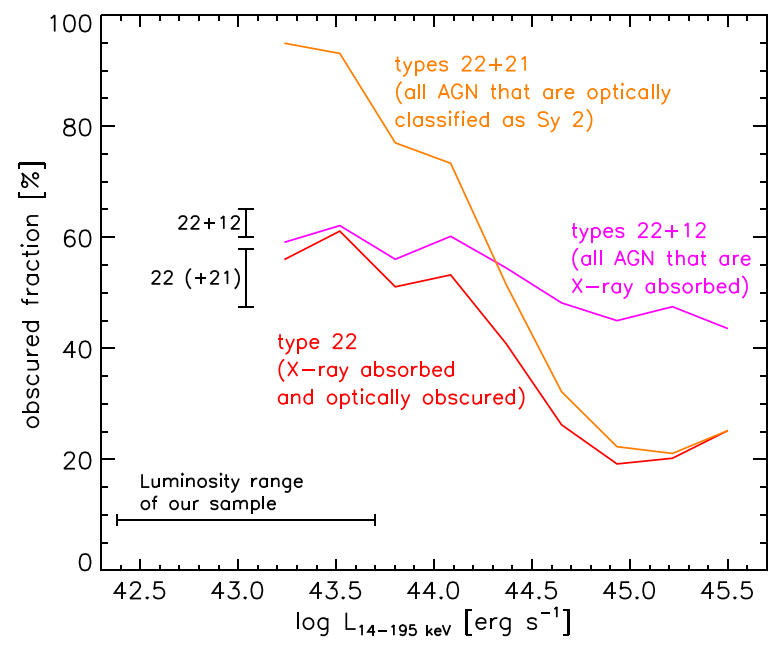

Figure 7. Fractions of optically and X-ray obscured AGNs as a function of luminosity. The curves are adapted from Figure 12 of Merloni et al. (2014). The details of the types, defined by those authors, are summarized in Section 4.3: the first/second digit of the type codes for optical/X-ray obscuration. The luminosity scale has been derived from $L_{2-10}^{\text {int }}$ as indicated in Section 3. The black ranges refer to our sample, and indicate the impact of allowing some flexibility in the definition of optically obscured and X-ray absorbed. At overlapping luminosities, the sample have similar fractions of AGNs that are X-ray absorbed (types 22+12) and that are both X-ray absorbed and optically obscured (type 22); but the fractions of AGNs classified as Sy 2 (types $22+21$ ) are very different.

out 4 for a variety of reasons, and confirmed only 3 as unabsorbed Sy 2s. Using a variety of metrics for a critical assessment of 24 objects that were previously claimed to be unabsorbed Sy 2s (again including many from Panessa \& Bassani 2002), Shi et al. (2010) could confirm only two as genuine, with broad emission lines 2-3 orders of magnitude fainter than typical Sy 1s-although several more candidates may have anomalously weak broad lines, this could not be confirmed with existing data. These authors concluded that $1 \%$ or less of Sy 2s are X-ray unabsorbed.

In contrast, a much higher fraction of such sources- $30 \%$ of AGNs at $\log L_{14-195} \sim 43$ - appears in the Merloni et al. (2014) sample. Whether this indicates that there is a large population of X-ray unabsorbed Sy $2 \mathrm{~s}$ or is due to a classification bias was discussed in depth by these authors. With reference to stacked optical spectra and spectral energy distributions, they suggested that many of the lower luminosity AGNs may have been incorrectly photometrically classified as Sy 2 due to the low contrast of the AGNs against the relatively bright host galaxy. On the other hand, spectroscopic data are sensitive to features associated with Sy 1s, such as broad lines, even when they are weak. Such signatures would not be evident in broad-band integrated photometry. This difference is reflected in their data: while $77 \%$ of the AGNs with only integrated broad-band photometric data are classified as Sy 2, only 56\% of AGNs with spectroscopic data are classified as Sy 2 s. The lower total fraction of Sy $2 \mathrm{~s}$ based on spectroscopic classifications implies in turn a much lower fraction of X-ray unabsorbed Sy 2 s.

Figure 6 shows that in our volume limited sample of Swift$B A T$ AGNs, there are no sources classified as X-ray unabsorbed Sy 2, implying these sources are rare. In this context, a simulation of the AGN population can provide valuable insight, since we know the Sy 2 fractions in both flux limited and volume limited samples. We describe this simulation in Section 4.3, aiming to answer the question of which curve from Merloni et al. (2014) in Figure 7 properly traces the fraction of Sy $2 \mathrm{~s}$ : is it types $22+21$ or just type 22 ?

\subsection{A Simulated Swift-BAT sample}

Our aim here is to simulate parent populations of AGNs with different prescriptions for the intrinsic fraction of Sy $2 \mathrm{~s}$. After applying observational limits, we can then use constraints from the flux-limited sample of Winter et al. (2009) and our own volume-limited sample to discriminate between the different prescriptions, giving us a handle on the true fraction of Sy 2 s.

We begin by constructing a population of AGNs using a 2-10 keV luminosity function from Aird et al. (2010) suitable for low redshift:

$$
\phi\left(L_{2-10}^{\mathrm{int}}\right) \propto\left[\left(L_{2-10}^{\mathrm{int}} / L_{0}\right)^{0.70}+\left(L_{2-10}^{\mathrm{int}} / L_{0}\right)^{3.14}\right]^{-1}
$$

where $\log L_{0}=44.96$. We have then randomly assigned each AGN to be a Sy 1 or Sy 2 according to the obscured fraction as a function of luminosity. We use two functions, which are discussed below. We allow the AGNs to be observed by calculating $L_{14-195}$ from $L_{2-10}$, assigning them random locations in a local volume, deriving their fluxes, and assessing whether they would be detected in the Swift-BAT survey. In doing this, we have not included the effects of X-ray absorption in the 14-195 keV band since, as we have seen, it has little impact at such high energies for the absorbing columns expected. We use a limit appropriate for the 9 months survey in order to select a flux limited sample matching that used by Winter et al. (2009); and a limit appropriate for the 58 months survey to match our volume limited selection outlined in this paper.

The left panels in Figure 8 show the two obscuration functions used in our simulations. The dotted line in the upper left panel is taken directly from Merloni et al. (2014) and was designed to follow the curve for types $22+21$; the dashed line in the lower left panel is our modification of this function to match the type 22 data, those AGNs that are both optically obscured and X-ray absorbed. The results of our simulations using these functions are also given in Figure 8. The center 

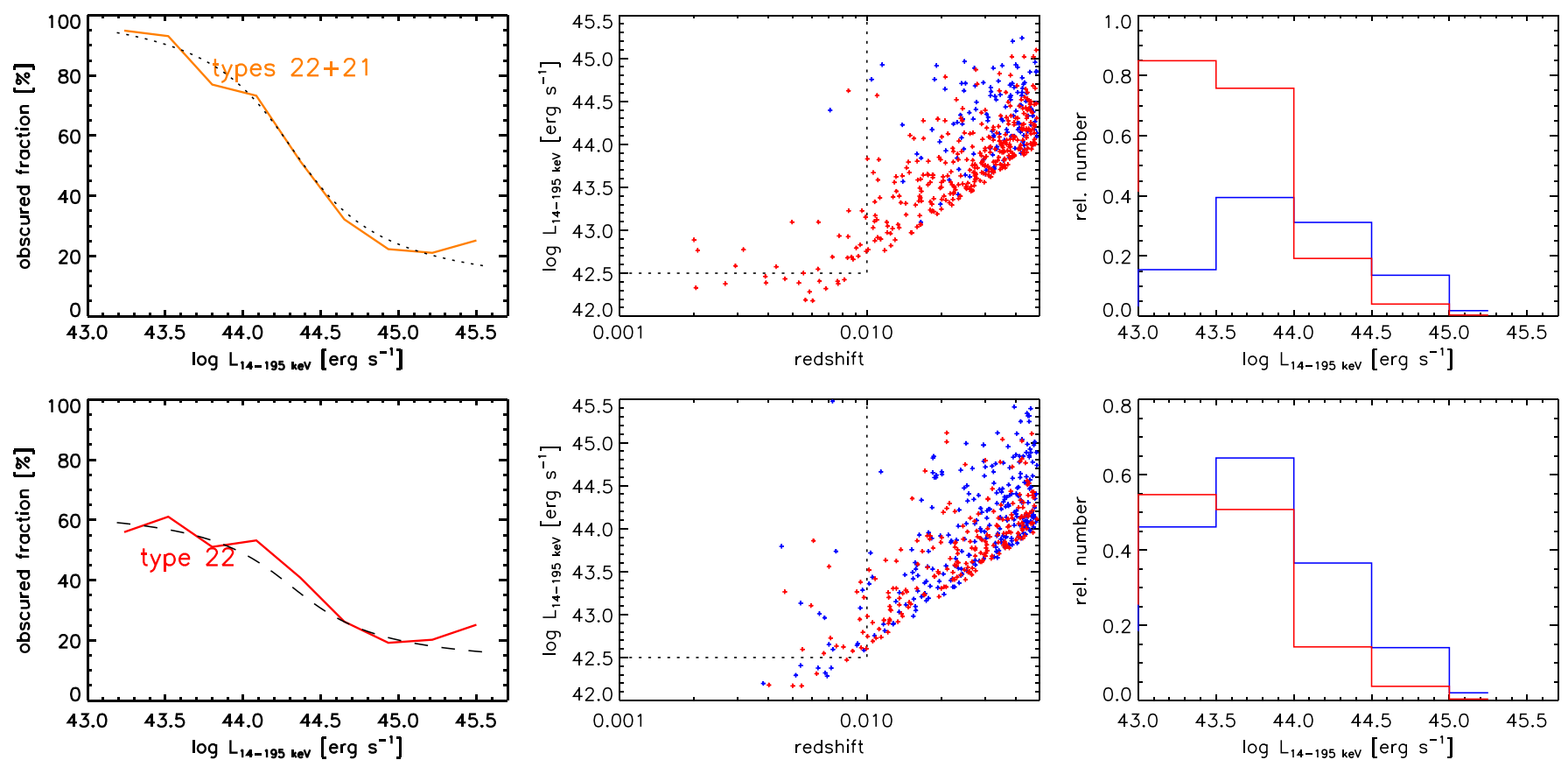

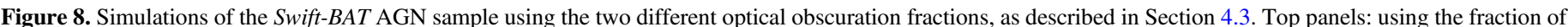

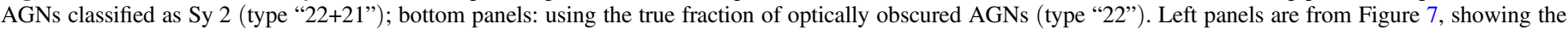

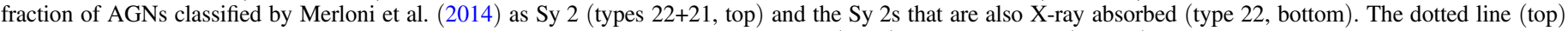

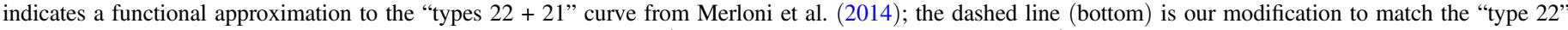

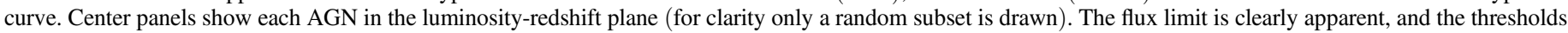

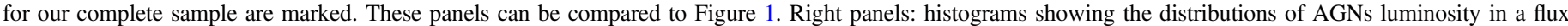

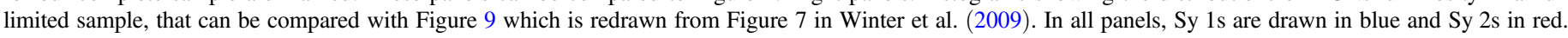

panels show the Sy 1 and Sy 2 populations generated-which can be compared to the real data in Figure 1. The right panels show their resulting distributions as a function of luminosity, which can be compared to Figure 9. In these panels, the sharp decrease in the number of Sy $2 \mathrm{~s}$ around $\log L_{14-195} \sim 44$ simply reflects the rapid change in the fraction of obscured AGNs at this luminosity. The flux limit means that low luminosity AGNs are not detected at larger distance, and hence biasses the apparent fraction of obscured AGNs at $\log L_{14-195}<43.5$. In contrast, because our sample is complete to $\log L_{14-195}=42.5$, it provides an unbiassed indication of the obscured fraction at these luminosities.

It is immediately clear that the lower panels better match the observed distribution of Sy 1 and Sy 2 sources reported in Winter et al. (2009). Similarly, the lower panels correspond to finding a fraction of $54 \%$ of Sy 2 s (in contrast to $87 \%$ for the upper panels) in a complete volume limited sample equivalent to that described in Section 2, again providing a good match to the fraction actually measured in the Swift$B A T$ sample.

Our conclusion is that, over the luminosity range $43<$ $\log L_{14-195}<44.5$ where the "type 22" and "types $22+21$ " curves differ in Figure 7, the true fraction of Sy 2s is given by the "type 22" curve. This confirms that X-ray unabsorbed Sy 2 s are rare, at most a few percent of the Sy 2 population; and that the high number of type 21 objects in the Merloni et al. (2014) sample is due to a bias in photometric classification. It also implies that, in low luminosity systems, optical obscuration and X-ray absorption are usually found together-and hence most likely originate in the same shared obscuring structure.

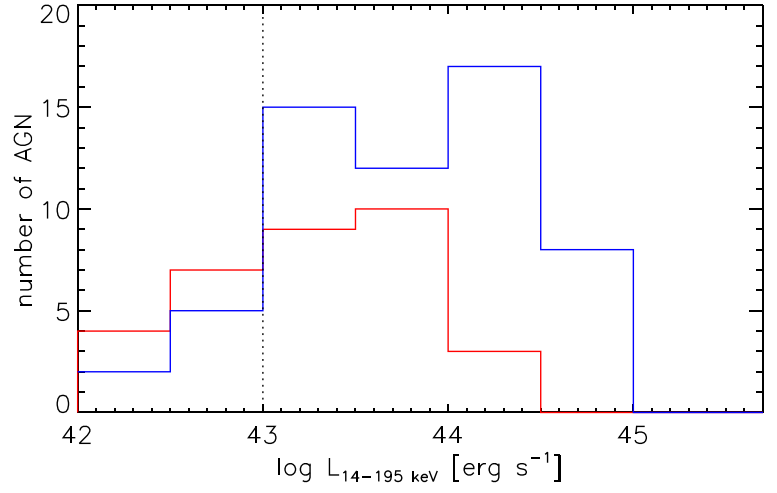

Figure 9. Number of Sy 1s (blue) and Sy 2s (red) in the flux limited sample of Winter et al. (2009). This figure is redrawn from their Figure 7 using data from their Tables 1 and 4, but plotted as a function of $14-195 \mathrm{keV}$ luminosity derived from $L_{2-10}^{\mathrm{int}}$ as indicated in Section 3 . The luminosity range to the right of the dotted line is that discussed in this paper.

\section{THE TORUS AND BLR}

Figure 7 shows that the behavior of the Sy 2 fraction and $\mathrm{X}$-ray absorbed fraction of AGNs are different above and below a luminosity of $\log L_{14-195} \sim 44$. In this section we explore what this may indicate about the torus. Often, the torus is simply considered to be a dusty entity, since it must obscure the BLR in some AGNs. A more careful definition would be that the torus is a geometrically thick structure (since it confines the ionization cone and any outflow) that causes optical obscuration and some (perhaps much) of the cold X-ray absorption. We show that, in this case, its inner boundary is decoupled from the dust sublimation radius. Thus, while nearinfrared reverberation mapping (Suganuma et al. 2006) and 


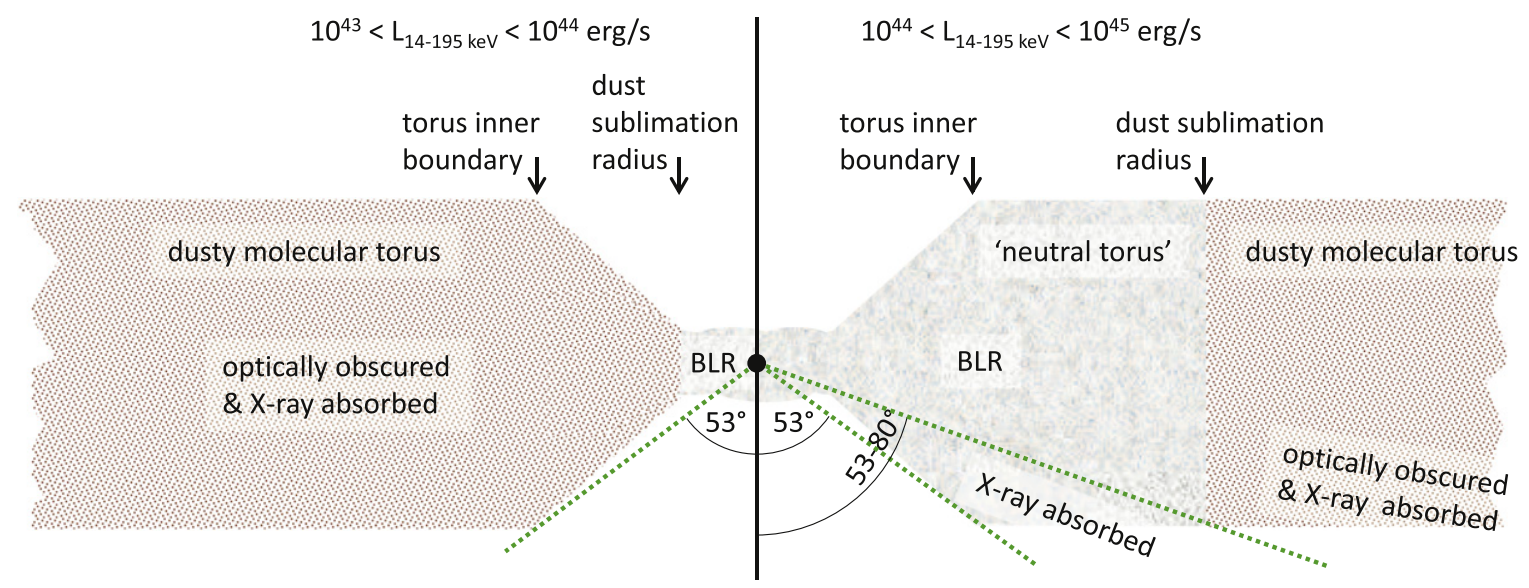

Figure 10. Cartoon illustrating how the inner edge of the torus and the dust sublimation radius $R_{\text {dust }}$ are related, depending on luminosity (we do not distinguish smooth or clumpy torus concepts here, and the cartoon could also be envisaged as a less precisely demarcated cloud distribution). At the luminosity range on the left side, the fraction of optically obscured and X-ray absorbed AGNs is the same. Because $R_{\text {dust }}$ is smaller than the radius of the inner edge of the geometrically thick dusty torus, it has no impact on the obscured fraction (which is set by the height of the thick structure). The right side depicts the situation at higher luminosities. Here, one finds essentially the same geometrically thick gas structure, but now $R_{\text {dust }}$ is larger than the radius of its inner wall. Thus the broad-line region extends outward into the neutral torus which, further out still, becomes the dusty torus. At these luminosities, the fraction of X-ray absorbed AGNs is the same, but the optically obscured fraction decreases with increasing luminosity.

interferometric data (Kishimoto et al. 2009, 2011; Weigelt et al. 2012) measure the dust sublimation radius, they do not measure the inner boundary of the torus.

\subsection{At Low Luminosity, $\log L_{14-195}<44$}

At luminosities below $\log L_{14-195} \sim 44$, the optically obscured AGN fraction of $\sim 60 \%$ is similar to the X-ray absorbed fraction, and is independent of luminosity. Since the dust sublimation radius $R_{\text {dust }}$ increases with luminosity, this independence must reflect the geometrical properties of the torus which we can constrain as follows. A luminosity of $\log L_{14-195}=44$ implies $R_{\text {dust, } 44} \sim 0.2 \mathrm{pc}$ for graphite grains of size $0.05 \mu \mathrm{m}$ (Netzer 2014). An obscured fraction of $60 \%$ implies $H / R \sim 0.75$ - a half opening angle of $\sim 53^{\circ}$-and hence $H_{\text {dust } 44} \sim 0.15 \mathrm{pc}$. And that the obscured fraction is constant with luminosity implies that $H / R$ cannot increase at radii smaller than $R_{\text {dust, } 44}$ (and the rapid drop in the optically obscured fraction at higher luminosities indicates that $H / R$ also decreases at larger radii out to a few times $R_{\text {dust,44). This is }}$ illustrated in the left side of Figure 10. There are two implications. First, it is the same geometrically thick structure that causes the optical obscuration and the X-ray absorption, and this structure is synonymous with the dusty torus. The implied half-opening angle of $\sim 53^{\circ}$ is at the upper end of the $30^{\circ}-50^{\circ}$ range derived from $3 \mathrm{D}$ models of the ionization cone kinematics (Müller-Sánchez et al. 2011; Fischer et al. 2013), a marginal consistency that could reflect uncertainties in the derivations of these numbers or may have a more physical origin. Second, the inner wall of the dusty torus-which defines the optically obscured and X-ray absorbed fractionshas universal properties at $\log L_{14-195}<44$ : the average torus properties are an inner radius of $\sim 0.2 \mathrm{pc}$ and a scale height of $\sim 0.15 \mathrm{pc}$, independent of luminosity. Here we make two notes. The first is that this is a constraint on the geometrically thick nature of the torus, and any dust that exists at smaller radii will not contribute to the obscured fraction because it is not driving the maximum value of $H / R$ (Netzer \& Laor 1993; Netzer 2014). The second is that at luminosities below that of our sample, one would expect the torus properties to change if there is a luminosity threshold below which the torus disappears (Elitzur \& Shlosman 2006).

\subsection{At High Luminosity, $\log L_{14-195}>44$}

While none of the AGNs in our sample are this luminous, the data of Merloni et al. (2014), as reproduced in Figure 7, show a dramatic difference in the Sy 2 and X-ray absorbed fractions. At these luminosities the Sy 2 fraction is not expected to suffer a significant bias due to mis-classification simply because the AGNs are brighter with respect to the host galaxy; indeed, the fraction of AGNs classified as type 21 by Merloni et al. (2014) does decrease dramatically. As such, one can consider the Sy 2 fraction as the optically obscured fraction. This fraction drops rapidly to $\sim 20 \%$ following the $L^{0.5}$ dependence of the dust sublimation radius, as understood in the "receding torus" concept (Lawrence 1991; Simpson 1998, 2005; Lusso et al. 2014), without requiring any change in the scale height of the torus. In contrast, the X-ray absorbed fraction remains nearly constant, falling only from $60 \%$ to $45 \%$.

The implicit population of X-ray absorbed Sy 1s were discussed by Merloni et al. (2014), who argued that the X-ray absorption is not occuring on large galaxy-wide scales and suggested instead that it is more plausibly due to dust-free gas within the BLR. Below, we argue that this is indeed likely, since BLR clouds are expected to contain a significant column of neutral gas; and associate the absorption with a "neutral torus" that co-exists with the BLR, and which has an impact on how the "receding torus" concept should be understood.

However, first it is important to clarify whether warm absorbers, which have been observed in about $50 \%$ of Sy $1 \mathrm{~s}$ (Komossa 1999; Blustin et al. 2005; Winter et al. 2012; Laha et al. 2014), may be responsible for the X-ray absorbed Sy 1s here. Warm absorbers imprint their signature on an X-ray spectrum through absorption lines and are most prominent in soft X-ray bands. They are classically identified by the $\mathrm{O}$ VII and $\mathrm{O}$ VIII absorption edges at 0.74 and $0.87 \mathrm{keV}$, respectively, rather than through their impact on the spectral shape (Komossa 1999; Winter et al. 2012; Laha et al. 2014). Warm absorbers have a smaller impact than neutral gas on the global 
shape of the spectrum, as evident from the models of Page et al. (2011) whose fits to $0.2-10 \mathrm{keV}$ QSO spectra show that a warm absorber requires an order of magnitude more gas than a neutral absorber to reproduce the same spectral shape. The median ionized column derived by Winter et al. (2012) for the Swift-BAT AGNs, as well as by Laha et al. (2014) on a different sample, is $\sim 10^{21} \mathrm{~cm}^{-2}$, with columns an order of magnitude lower for AGNs without strong $\mathrm{O}$ VII and $\mathrm{O}$ vIII detections. While significant, this is less than $\sim 10^{21.5} \mathrm{~cm}^{-2}$ threshold adopted here for defining X-ray absorbed systems. Coupled with the fact that Merloni et al. (2014) used either the full $0.5-10 \mathrm{keV}$ band or the $0.5-2 \mathrm{keV}$ versus $2-10 \mathrm{keV}$ hardness ratio to derive the absorbing column from its effect across the whole band rather than in specific features, this strongly argues that the absorptions they derived are due to neutral rather than ionized gas.

The BLR provides an ample source of neutral gas that could be responsible for the derived absorption. Photoionization models by Netzer (2013) show that for a typical BLR ionization parameter of $U \sim 0.01$ (Leighly \& Casebeer 2007; Negrete et al. 2013) and a density of $10^{10} \mathrm{~cm}^{-3}$ the temperature drops below $10^{4} \mathrm{~K}$ at a column of $\sim 10^{21} \mathrm{~cm}^{-2}$ and the fractional abundance of $\mathrm{H}_{\mathrm{I}}$ increases dramatically. Thus, for clouds with a column density of $10^{23} \mathrm{~cm}^{-2}$, as typically expected, the majority of gas is neutral rather than ionized. This has been used to explain the cause of Sy 1s which change state on short timescales, in terms of a BLR cloud passing across the line of sight to the central engine (see Risaliti et al. 2010 and Torricelli-Ciamponi et al. 2014, and references therein). In this interpretation, the clouds have cores with column densities of at least a few $\times 10^{23} \mathrm{~cm}^{-2}$ that cause the neutral absorption, and ionized tails that are the origin of the warm absorption (Risaliti et al. 2009, 2011).

Since the BLR clouds contain abundant neutral gas, it is natural to associate them with the X-ray absorbed Sy 1s. The remarkable constancy of the fraction of X-ray absorbed AGNs across the luminosity range in Figure 7 suggests that the geometry of the cold gas absorber also changes rather little: both the inner edge and scale height of the thick gas distribution remain similar to those at low luminosities. The implication is that there is a "neutral torus" that co-exists with the BLR, and extends out to the start of the dusty molecular torus as illustrated in the right-hand side of Figure 10. In this context, we note that Minezaki \& Matsushita (2015) and Gandhi et al. (2015) have independently suggested the core of the fluorescent $\mathrm{Fe} \mathrm{K} \alpha$ line may originate at radii extending from the BLR out to the dusty torus, implying the presence of gas at intermediate radii. There is no clear boundary between the BLR and the neutral torus since it is the same clouds that contribute to both. The BLR emission will follow a radial dependence associated with the ionization parameter. Since $U \propto L_{\mathrm{AGN}} /\left(R^{2} n_{\mathrm{H}}\right)$, the rapid decrease of $U$ with radius $R$ may be largely mitigated if the cloud density $n_{\mathrm{H}}$ also decreases with $R$. Thus the BLR could extend far into the neutral torus. How far it extends is given by the scaling of the BLR size with AGN luminosity (Kaspi et al. 2000). Using the most recent measurement of that relation (Bentz et al. 2013), and adopting a ratio $L_{\mathrm{AGN}} / \lambda L_{5100} \sim 15$ (Grupe et al. 2004), we estimate the characteristic radius of the BLR to be a factor of a few less than the dust sublimation radius. However, it is also possible that the BLR emission could still occur as far as the dust sublimation radius.
In the context above, the idea of the "receding torus," in which the location of the inner wall of the torus is set by the dust sublimation radius, can be misleading. We argue that the inner dust boundary and the inner gas boundary of the torus should be considered separately. The discussion above suggests that the location of the inner gas boundary of the geometrically thick structure (causing the X-ray absorption), is roughly independent of AGN luminosity in the range $43<\log L_{14-195}<45$. And it is within this geometrically thick gas structure that the inner dust boundary (i.e., the "receding torus") simply represents the location at which the predominant gas phase changes from ionized/neutral to dusty/molecular. The thick gas structure itself remains nearly unchanged. This means that at low luminosities, the thick gas structure is synonymous with the standard dusty torus; but at high luminosities, the thick gas structure has an outer part that is the dusty torus, as well as an inner part that contributes to the BLR emission but also acts as a neutral dust-free torus.

Intriguingly, Winter et al. (2012) found that the strength of the $\mathrm{O}$ VII and $\mathrm{O}$ VIII edges tracing the warm absorbers in Swift$B A T$ AGNs are correlated with the neutral column densitysuggesting that material in the neutral torus is associated with a more-or-less proportional amount of ionized material. This can be put in the context of AGNs showing rapid variations in absorbing column, in which neutral clouds have an ionized outflowing tail (Risaliti et al. 2009, 2011) —an interpretation that applies to individual clouds in the BLR. Given that warm absorbers are outflowing (Blustin et al. 2005; Laha et al. 2014), and that the distance of the warm absorbers from the AGN is typically larger than the BLR but within a factor of a few of the dust sublimation radius (Blustin et al. 2005), one might speculate that the ionized material seen by Winter et al. (2012) is associated with the neutral torus and outflowing from the clouds as they are ablated.

\section{CONCLUSIONS}

We have described the rationale for defining a complete volume limited sample of bright local Seyferts, selected from the 14-195 keV Swift-BAT catalog. These AGNs are complemented by a matched sample of inactive galaxies, and we have shown that the host galaxy properties (stellar mass, morphological type, inclination, presence of a bar, distance) of these two samples exhibit similar distributions. Spatially and spectrally resolved observations of the inner few hundred parsecs in these active and inactive galaxies will be used to study gas inflow and outflow and the processes regulating it. The main points we have discussed here are the following.

1. A comparison of the properties of the Sy $1 \mathrm{~s}$ and Sy $2 \mathrm{~s}$ shows that there is no bias for type in the selection. The only significant difference in AGN properties is the absorbing column $N_{\mathrm{H}}$, the mean of which is two orders of magnitude greater for the Sy $2 \mathrm{~s}$. This is consistent with unification schemes in which optical obscuration is greater for Sy 2 s, but cannot distinguish between simple orientation dependence and instrinsic differences in torus properties.

2. The fraction of Sy 1 s versus Sy 2 s in this sample, and also in the flux limited $14-195 \mathrm{keV}$ sample of Winter et al. (2009), demonstrates that the true fraction of Sy $2 \mathrm{~s}$ at $\log L_{14-195} \sim 42.5-44$ is $50 \%-60 \%$, consistent with Lawrence \& Elvis (2010) and the spectroscopic 
subsample of Merloni et al. (2014). We show that the fraction of X-ray unabsorbed Sy $2 \mathrm{~s}$ is at most a few percent and hence that, at these luminosities, optical obscuration and X-ray absorption usually occur together.

3. At higher luminosities $\log L_{14-195}>44$, while the optically obscured fraction drops rapidly, the X-ray absorbed fraction remains the same. We argue this implies that the inner boundary of the geometrically thick gas structure associated with the torus is roughly independent of AGN luminosity, having similar radial and height scales as for lower luminosities. At low luminosities this gas structure is synonymous with the standard dusty torus; but at high luminosities it has an outer part which is the dusty torus, and an inner part which is a neutral dust-free torus that also contributes to the BLR emission. In this context, the "receding torus" model simply represents the location within this gas structure at which the predominant phase changes from ionized/neutral to molecular/dusty.

4. Finally, we note that the consistency of local (e.g., Swift$B A T$ ) and more distant (e.g., the spectroscopically classified AGNs in the $0.3<z<3.5$ sample of Merloni et al. 2014) samples suggests that the obscuring structure does not strongly evolve with redshift.

The authors thank the referee for a variety of useful suggestions that have helped improve the paper. They are also grateful to $\mathrm{K}$. Dodds-Eden for her major contribution to selecting the matched inactive sample. R.D. thanks A. Merloni and H. Netzer for useful discussions. E.K.S.H. acknowledges support from the NSF Astronomy and Astrophysics Research Grant under award AST-1008042. C.R. acknowledges financial support from the CONICYT-Chile "EMBIGGEN" Anillo (grant ACT1101). R.R. thanks CNPq for financial support. M.K. acknowledges support from the Swiss National Science Foundation (SNSF) through the Ambizione fellowship grant PZ00P2 154799/1. This research has made use of the NASA/ IPAC Extragalactic Database (NED) which is operated by the Jet Propulsion Laboratory, California Institute of Technology, under contract with the National Aeronautics and Space Administration. It also makes use of data products from the 2MASS, which is a joint project of the University of Massachusetts and the Infrared Processing and Analysis Center/California Institute of Technology, funded by the National Aeronautics and Space Administration and the National Science Foundation.

\section{REFERENCES}

Aird, J., Nandra, K., Laird, E. S., et al. 2010, MNRAS, 401, 2531 Alexander, D., Stern, D., Del Moro, A., et al. 2013, ApJ, 773, 125

Asmus, D., Gandhi, P., Smette, A., Hönig, S., \& Duschl, W. 2012, A\&A, 536, 36

Asmus, D., Hönig, S., Gandhi, P., Smette, A., \& Duschl, W. 2014, MNRAS, 439, 1648

Bauer, F., Arévalo, P., Walton, D., et al. 2014, ApJ, submitted (arXiv:1411.0670)

Baumgartner, W., Tueller, J., Markwardt, C., et al. 2013, ApJSS, 207, 19

Barbosa, F., Storchi-Bergmann, T., Cid Fernandes, R., Winge, C., \& Schmitt, H. 2006, MNRAS, 371, 170

Barbosa, F., Storchi-Bergmann, T., Cid Fernandes, R., Winge, C., \& Schmitt, H. 2009, MNRAS, 396, 2

Beckmann, V., Jean, P., Lubiński, P., Soldi, S., \& Terrier, R. 2011, A\&A, 513,70

Benson, A., Bower, R., Frenk, C., et al. 2003, ApJ, 599, 38

Bentz, M., Denney, K. D., Grier, C. J., et al. 2013, ApJ, 767, 149
Bian, W., \& Gu, Q. 2007, ApJ, 657, 159

Bianchi, S., Panessa, F., Barcons, X., et al. 2012, MNRAS, 426, 3225

Bianchi, S., Pioncelli, E., Chiaberge, M., et al. 2009, ApJ, 695, 781

Blustin, A., Page, M., Fuerst, S., Branduardi-Raymont, G., \& Ashton, C. 2005, A\&A, 431, 111

Bonnet, H., Abuter, R., Baker, A., et al. 2004, The ESO Messenger, 117, 17 Brightman, M., \& Nandra, K. 2008, MNRAS, 390, 1241

Brightman, M., \& Nandra, K. 2011, MNRAS, 413, 1206

Burtscher, L., Orban de Xivry, G., Davies, R., et al. 2015, A\&A, in press (arXiv:1504.01104)

Croton, D., Springel, V., White, S. D. M., et al. 2006, MNRAS, 365, 11

Davies, R., Burtscher, L., Dodds-Eden, K., \& Orban de Xivry, G. 2012, JPhCS, 372, 012046

Davies, R., Müller Sánchez, F., Genzel, R., et al. 2007, ApJ, 671, 1388

Davies, R., Maciejewski, W., Hicks, E. K. S., et al. 2014, ApJ, 792, 101

de Vaucouleurs, G., de Vaucouleurs, A., Corwin, H., et al. 1991, The Third Reference Catalogue of Bright Galaxies (RC3) (New York: Springer)

Diamond-Stanic, A., Rieke, G., \& Rigby, J. 2009, ApJ, 698, 623

Dumas, G., Mundell, C., Emsellem, E., \& Nagar, N. 2007, MNRAS, 379, 1249

Eisenhauer, F., Abuter, R., Bickert, K., et al. 2003, in Proc. SPIE, 4841, 1548

Elitzur, M., \& Ho, L. 2009, ApJL, 701, L91

Elitzur, M., \& Shlosman, I. 2006, ApJL, 648, L101

Elvis, M., Risaliti, G., Nicastro, F., et al. 2004, ApJ, 615L, 25

Evans, D., Lee, J., Turner, J., Weaver, K., \& Marshall, H. 2007, ApJ, 671, 1345

Fabian, A. 2012, ARA\&A, 50, 455

Feltre, A., Hatziminaoglou, E., Fritz, J., \& Franceschini, A. 2012, MNRAS, 426, 120

Fischer, T., Crenshaw, D., Kraemer, S., \& Schmitt, H. 2013, ApJSS, 209, 1

Gandhi, P., Horst, H., Smette, A., et al. 2010, in AIP Conf. Proc. 1248, X-ray Astronomy 2009: Present Status Multiwavelength Approach and Future Perspectives, 431

Gandhi, P., Hönig, S., \& Kishimoto, M. 2015, ApJL, submitted (arXiv:1502.02661)

Garciá-Burillo, S. 2011, in Astronomy at High Angular Resolution 2011: The Central Kiloparsec in Galactic Nuclei (Bristol: IOPP)

Gliozzi, M., Sambruna, R., \& Foschini, L. 2007, ApJ, 662, 878

Grupe, D., Wills, B., Leighly, K., \& Meusinger, H. 2004, AJ, 127, 156

Gu, Q., \& Huang, J. 2002, ApJ, 579, 205

Haan, S., Schinnerer, E., Emsellem, E., et al. 2009, ApJ, 92, 1623

Harris, G., Rejkuba, M., \& Harris, W. 2010, PASA, 27, 457

Hawkins, M. 2004, A\&A, 519, 529

Heisler, C., Lumsden, S., \& Bailey, J. 1997, Natur, 385, 700

Hickox, R., Mullaney, J., Alexander, D., et al. 2014, ApJ, 782, 9

Hicks, E., Davies, R., Maciejewski, W., et al. 2013, ApJ, 768, 107

Hicks, E., Davies, R., Malkan, M., et al. 2009, ApJ, 696, 448

Ho, L. 2008, ARA\&A, 46, 475

Hönig, S. 2013, in Proc. of the Torus Workshop 2012, ed. C. Packham, R. Mason, \& A. Alonso-Herrero (San Antonio, TX: UTSA), 157

Hopkins, P., \& Quataert, E. 2010, MNRAS, 407, 1529

Ichikawa, K., Packham, C., Ramos Almeida, C., et al. 2015, ApJ, 803, 57

Karouzos, M., Jarvis, M., \& Bonfield, D. 2014, MNRAS, 439, 861

Kaspi, S., Smith, P., Netzer, H., et al. 2000, ApJ, 533, 631

Kauffmann, G., Heckman, T. M., White, S. D. M., et al. 2003, MNRAS, 341,33

Kishimoto, M., Hönig, S., Antonucci, R., et al. 2009, A\&A, 507, L57

Kishimoto, M., Hönig, S., Antonucci, R., et al. 2011, A\&A, 527, 121

Kocevski, D., Faber, S. M., Mozena, M., et al. 2012, ApJ, 744, 148

Komossa, S. 1999, in ASCA/ROSAT Workshop on AGN and the X-ray Background, ISAS Report, ed. T. Takahashi, \& H. Inoue (arXiv:astro-ph/ 0001263)

Kormendy, J., \& Ho, L. 2013, ARA\&A, 51, 511

Koss, M., Mushotzky, R., Baumgartner, W., et al. 2013, ApJL, 765, L26

Koss, M., Mushotzky, R., Veilleux, S., \& Winter, L. 2010, ApJL, 716, L125

Koss, M., Mushotzky, R., Veilleux, S., et al. 2011, ApJ, 739, 57

Laha, S., Guainazzi, M., Dewangan, G., Chakravorty, S., \& Kembhavi, A. 2014, MNRAS, 441, 2613

Laor, A. 2003, ApJ, 590, 86

Lawrence, A. 1991, MNRAS, 252, 586

Lawrence, A., \& Elvis, M. 2010, ApJ, 714, 561

Leighly, K., \& Casebeer, D. 2007, in ASP Conf. Ser. 373, The Central Engine of Active Galactic Nuclei, ed. L. Ho, \& J.-M. Wang (San Francisco, CA: ASP), 365

Lusso, E., Hennawi, J., Comastri, A., et al. 2014, ApJ, 777, 86

Marinucci, A., Bianchi, S., Nicastro, F., Matt, G., \& Goulding, A. 2012, ApJ, 748,130

Matt, G., Guainazzi, M., Frontera, F., et al. 1997, A\&A, 325, L13 
Matt, G., Pompilio, F., \& La Franca, F. 1999, NewA, 4, 191

Mazzalay, X., Saglia, R., Erwin, P., et al. 2013, MNRAS, 428, 2389

Meléndez, M., Kraemer, S., Armentrout, B., et al. 2008, ApJ, 682, 94

Meléndez, M., Mushotzky, R., Shimizu, T., Barger, A., \& Cowie, L. 2014, ApJ, 794, 152

Merloni, A., Bongiorno, A., Brusa, M., et al. 2014, MNRAS, 437, 3550

Minezaki, T., \& Matsushita, K. 2015, ApJ, 802, 98

Müller-Sánchez, F., Davies, R., Genzel, R., et al. 2009, ApJ, 691, 749

Müller-Sánchez, F., Prieto, M. A., Hicks, E., et al. 2011, ApJ, 739, 69

Mushotzky, R., Shimizu, T., Meléndez, M., \& Koss, M. 2014, ApJL, 781, L34

Negrete, C., Dultzin, D., Marziani, P., \& Sulentic, J. 2013, ApJ, 771, 31

Netzer, H. 2013, The Physics and Evolution of Active Galactic Nuclei (New York: Cornell Univ. Press)

Netzer, H. 2015, ARA\&A, vol. 53 arXiv:1505.00811

Netzer, H., \& Laor, A. 1993, ApJL, 404, L51

Nicastro, F. 2000, ApJL, 530, L65

Novak, G., Ostriker, J., \& Ciotti, L. 2011, ApJ, 737, 26

Page, M., Carrera, F., Stevens, J., Ebrero, J., \& Blustin, A. 2011, MNRAS, 416, 2792

Panessa, F., \& Bassani, L. 2002, A\&A, 394, 435

Pappa, A., Georgantopoulos, I., Stewart, G., \& Zezas, A. 2001, MNRAS, 326, 995

Ramos Almeida, C., Levenson, N., Alonso-Herrero, A., et al. 2011, ApJ, 731,92

Reunanen, J., Kotilainen, J., \& Prieto, M. A. 2003, MNRAS, 343, 192

Risaliti, G., Maiolino, R., \& Salvati, M. 1999, ApJ, 522, 157

Risaliti, G., Salvati, M., Elvis, M., et al. 2009, MNRAS, 393, 1

Risaliti, G., Elvis, M., Bianchi, S., \& Matt, G. 2010, MNRAS, 496, L20

Risaliti, G., Nardini, E., Salvati, M., et al. 2011, MNRAS, 410, 1027

Rupke, D., \& Veilleux, S. 2011, ApJL, 729, 27

Rupke, D., \& Veilleux, S. 2013, ApJL, 775, 15

Sakamoto, K., Okumura, S., Ishizuki, S., \& Scoville, N. 1999, ApJ, 525, 691

Schartmann, M., Burkert, A., Krause, M., et al. 2010, MNRAS, 403, 1801

Schawinski, K., Simmons, B., Urry, M., Treister, E., \& Glikman, E. 2012, MNRAS, 425, L61
Shi, Y., Rieke, G., Smith, P., et al. 2010, ApJ, 714, 115

Simpson, C. 1998, MNRAS, 297, L39

Simpson, C. 2005, MNRAS, 360, 565

Skrutskie, M., Cutri, R. M., Stiening, R., et al. 2006, AJ, 131, 1163

Smajić, S., Fischer, S., Zuther, J., \& Eckart, A. 2012, A\&A, 544, 105

Somerville, R., Hopkins, P., Cox, T., Brant, R., \& Hernquist, L. 2008, MNRAS, 391, 481

Sosa-Brito, R., Tacconi-Garman, L., Lehnert, M., \& Gallimore, J. 2001, ApJS, 136,61

Springel, V., di Matteo, T., \& Hernquist, L. 2005, MNRAS, 361, 776

Storchi-Bergmann, T. 2014, BrJPh, 43, 383

Storchi-Bergmann, T. 2015, in Proc. IAUS, Galaxies in 3D across the Universe, ed. B. Ziegler et al. (Cambridge: Cambridge Univ. Press), 190

Sturm, E., González-Alfonso, E., Veilleux, S., et al. 2011, ApJL, 733, L16

Suganuma, M., Yoshii, Y., Kobayashi, Y., et al. 2006, ApJ, 639, 46

Theureau, G., Hanski, M., Coudreau, N., Hallet, N., \& Martin, J.-M. 2007, A\&A, 465, 71

Torricelli-Ciamponi, G., Pietrini, P., Risaliti, G., \& Salvati, M. 2014, MNRAS, 442, 2116

Tran, H. 2001, ApJL, 554, L19

Tran, H. 2003, ApJ, 583, 632

Treister, E., Urry, M., \& Virani, S. 2009, ApJ, 696, 110

Vasudevan, R., Fabian, A., Gandhi, P., Winter, L., \& Mushotzky, R. 2010, MNRAS, 402, 1081

Veilleux, S., Melendez, M., Sturm, E., et al. 2013, ApJ, 778, 27

Vernet, J., Dekker, H., D’Odoric, S., et al. 2011, A\&A, 536, 105

Véron-Cetty, M.-P., \& Véron, P. 2010, A\&A, 518, 10

Villforth, C., Hamann, F., Rosario, D., et al. 2014, MNRAS, 439, 3342

Weaver, K., Meléndez, M., Mushotzky, R., et al. 2010, ApJ, 716, 1151

Weigelt, G., Hofmann, K.-H., Kishimoto, M., et al. 2012, A\&A, 541, L9

Westoby, P., Mundell, C., Nagar, N., et al. 2012, ApJSS, 199, 1

Winter, L., Lewis, K., Koss, M., et al. 2010, ApJ, 710, 503

Winter, L., Mushotzky, R., Reynolds, C., \& Tueller, J. 2009, ApJ, 690, 1322

Winter, L., Veilleux, S., McKernan, B., \& Kallman, T. 2012, ApJ, 745, 107

Yaqoob, T. 2012, MNRAS, 423, 3360 